\title{
A ROBUST MULTIGRID METHOD FOR THE TIME-DEPENDENT STOKES PROBLEM*
}

\author{
STEFAN TAKACS ${ }^{\dagger}$
}

\begin{abstract}
We propose a coupled multigrid method for generalized Stokes flow problems. Such problems occur as subproblems in implicit time-stepping approaches for time-dependent Stokes problems. The discretized Stokes system is a large-scale linear system whose condition number depends on the grid size of the spatial discretization and of the length of the time step. Recently, for this problem a coupled multigrid method has been proposed, where in each smoothing step a Poisson problem has to be solved (approximately) for the pressure field. In the present paper, we propose a coupled multigrid method where the solution of such sub-problems is not needed. We prove that the proposed method shows robust convergence behavior in the grid size of the spatial discretization and of the length of the time-step.
\end{abstract}

Key words. Generalized Stokes problem, coupled multigrid methods, robustness

AMS subject classifications. 65N55 65N22 76D07 65N30

1. Introduction. We consider the following model problem (generalized Stokes flow problem). Let $\Omega \subset \mathbb{R}^{2}$ be a bounded polygonal domain and assume $f \in\left[L^{2}(\Omega)\right]^{2}$ and $g \in L^{2}(\Omega)$ to be given. Find a velocity field $u$ and a pressure distribution $p$ such that

$$
\begin{aligned}
-\Delta u+\beta u+\nabla p & =f \text { in } \Omega, \\
\nabla \cdot u & =g \text { in } \Omega, \\
u & =0 \text { on } \partial \Omega
\end{aligned}
$$

is satisfied. $\beta>0$ is assumed to be a given parameter. The problem 1.1 appears as an auxiliary problem for implicit time-stepping approaches to solve an incompressible, time-dependent Stokes flow problem. In this case, the parameter $\beta$ is proportional to the inverse of the length of the time-step, scaled by a viscosity parameter.

To obtain existence and uniqueness of the solution, we further require $\int_{\Omega} p \mathrm{~d} x=$ $\int_{\Omega} g \mathrm{~d} x=0$. Note that the analysis presented in this paper is (due to the use of regularity results) restricted to convex domains. However, the numerical method presented in the paper can be applied also to non-convex domains.

The discretization of the problem leads to an indefinite linear system with saddlepoint structure. The main goal of this work is to construct and to analyze numerical methods that produce an approximate solution to the problem, where the computational complexity can be bounded by the number of unknowns times a constant which is independent of the grid level (of the spatial discretization) and the choice of $\beta$, in particular for large values of $\beta$ (which correspond to small time steps).

For the solution of such a saddle-point problem, there are several possibilities. In [5, 11, 13, 14, 16, 25], various kinds of preconditioners have been proposed for this problem which can be combined with a Krylov-subspace method as outer iteration scheme to yield an iterative solver for the problem.

An alternative is to apply a multigrid algorithm directly to the coupled system. On the one hand such methods are typically quite fast, on the other hand such an approach does not require an outer iteration scheme. For $\beta=0$, problem (1.1) is

\footnotetext{
${ }^{*}$ The research was funded by the Austrian Science Fund (FWF): J3362-N25.

${ }^{\dagger}$ Faculty of Mathematics, TU Chemnitz, Germany, (stefan.takacs@numa.uni-linz.ac.at)
} 
the standard (stationary) Stokes problem. For this case several multigrid solvers are available, see, e.g., [17, 21, 6, 4, 24] and the papers cited in [23, 15]. The construction of a multigrid method for $\beta>0$, particularly if the method is desired to show robust convergence behavior in $\beta$, is more involved, see [12] for an overview and numerical results. Recently, an important step forward has been archived by Olshanskii, who has proposed such a robust multigrid method, see 15. In the named paper however, a Poisson problem for the pressure has to be solved approximately (for example by applying one V-cycle, cf. Section 8 in [15) for each step of the smoothing iteration. (We will comment on this in Remarks 4.1 and 4.4).

The goal of the present paper is to drop this requirement and, therefore, reduce the computational costs. We propose a multigrid solver where the smoother is a simple linear iteration scheme and we prove that the proposed method is robust in the grid size of the spatial discretization and in the choice of $\beta$. We will present a convergence proof for our multigrid method based on the classical splitting of the analysis into smoothing property and approximation property, see [9].

The results of this paper form a basis of the convergence analysis of an all-at-once multigrid method for the Stokes optimal control problem, cf. [19.

This paper is organized as follows. In Section 2 we will introduce the variational formulation and discuss its discretization. The multigrid framework will be presented in Section 3. In Section 4 we will discuss the choice of the smoother. The proof of the approximation property will be given in Section 5. Numerical results which illustrate the convergence result will be presented in Section 6. In Section 7 we will close with conclusions.

2. Variational formulation and discretization. Here and in what follows, $L^{2}(\Omega)$ and $H^{1}(\Omega)$ denote the standard Lebesgue and Sobolev spaces with associated standard norms $\|\cdot\|_{L^{2}(\Omega)}$ and $\|\cdot\|_{H^{1}(\Omega)}$, respectively. The space $L_{0}^{2}(\Omega)$ is the space of all functions in $L^{2}(\Omega)$ with mean value 0 . The space $H_{0}^{1}(\Omega)$ is the space of all functions in $H^{1}(\Omega)$ that vanish on the boundary. Both spaces are equipped with standard norms, i.e., $\|\cdot\|_{L_{0}^{2}(\Omega)}:=\|\cdot\|_{L^{2}(\Omega)}$ and $\|\cdot\|_{H_{0}^{1}(\Omega)}:=\|\cdot\|_{H^{1}(\Omega)}$.

Using these spaces, we can set up the variational formulation of $(1.1)$, which reads as follows. Find $u \in U:=\left[H_{0}^{1}(\Omega)\right]^{2}$ and $p \in P:=L_{0}^{2}(\Omega)$ such that

$$
\begin{aligned}
(\nabla u, \nabla \tilde{u})_{L^{2}(\Omega)}+\beta(u, \tilde{u})_{L^{2}(\Omega)}+(p, \nabla \cdot \tilde{u})_{L^{2}(\Omega)} & =(f, \tilde{u})_{L^{2}(\Omega)} \\
(\nabla \cdot u, \tilde{p})_{L^{2}(\Omega)} & =(g, \tilde{p})_{L^{2}(\Omega)}
\end{aligned}
$$

holds for all $\tilde{u} \in U$ and $\tilde{p} \in P$. Certainly, the variational problem can be rewritten as one variational equation as follows. Find $x \in X$ such that

$$
\mathcal{B}(x, \tilde{x})=\mathcal{F}(\tilde{x}) \quad \text { for all } \tilde{x} \in X
$$

where $X:=U \times P, x:=(u, p), \tilde{x}:=(\tilde{u}, \tilde{p})$ and

$$
\begin{aligned}
\mathcal{B}((u, p),(\tilde{u}, \tilde{p})) & :=(\nabla u, \nabla \tilde{u})_{L^{2}(\Omega)}+\beta(u, \tilde{u})_{L^{2}(\Omega)}+(p, \nabla \cdot \tilde{u})_{L^{2}(\Omega)}+(\nabla \cdot u, \tilde{p})_{L^{2}(\Omega)} \\
\mathcal{F}(\tilde{u}, \tilde{p}) & :=(f, \tilde{u})_{L^{2}(\Omega)}+(g, \tilde{p})_{L^{2}(\Omega)} .
\end{aligned}
$$

We are interested in finding an approximative solution for equation (2.1). The convergence analysis follows standard approaches, i.e., we show that the problem in question is well posed in some norm $\|\cdot\|_{X}$ (which is for the Poisson problem the $H^{1}$ norm and for the standard Stokes problem the pair of $H^{1}$ for the velocity and $L^{2}$ for the pressure). In our case this norm will be parameter-dependent. In a second step, 
we will introduce a properly scaled $L^{2}$-like norm $\|\cdot\|_{k}$, which depends on the grid level $k$, see below. We will use an inverse inequality to show that $\|\cdot\|_{X} \leq C\|\| \cdot \|_{k}$ holds. The convergence of the multigrid method (smoothing property and approximation property) will be shown in the norm $\|\cdot \cdot\|_{k}$.

The main focus of the paper is the proof of the approximation property. Throughout the paper we will comment on the differences to the approach presented in [15], which allows us to drop the requirement of solving (approximately) Poisson problems. The key idea of the proof of the approximation property follows classical approaches. However, the way of constructing the norms is non-standard. For simplicity, we follow the abstract framework introduced in [20]. First we introduce the following convenient notation.

Notation 2.1. Throughout this paper, $C>0$ is a generic constant, independent of the grid level $k$ and the choice of the parameter $\beta$. For any scalars $a$ and $b$, we write $a \lesssim b($ or $b \gtrsim a)$ if there is a constant $C>0$ such that $a \leq C b$. We write $a \approx b$ if $a \lesssim b \lesssim a$.

Let the Hilbert spaces $X, U$ and $P$ (introduced above) be equipped with the following norms:

$$
\begin{aligned}
\|x\|_{X}^{2} & :=\|(u, p)\|_{X}^{2}:=\|u\|_{U}^{2}+\|p\|_{P}^{2}, \\
\|u\|_{U}^{2} & :=\|u\|_{H^{1}(\Omega)}^{2}+\beta\|u\|_{L^{2}(\Omega)}^{2} \text { and } \\
\|p\|_{P}^{2} & :=\sup _{0 \neq w \in\left[H_{0}^{1}(\Omega)\right]^{2}} \frac{(p, \nabla \cdot w)_{L^{2}(\Omega)}^{2}}{\|w\|_{H^{1}(\Omega)}^{2}+\beta\|w\|_{L^{2}(\Omega)}^{2}} .
\end{aligned}
$$

Lemma 2.1 in [15] states the following stability result.

LEMMA 2.2. The relation

$$
\|x\|_{X} \lesssim \sup _{0 \neq \tilde{x} \in X} \frac{\mathcal{B}(x, \tilde{x})}{\|\tilde{x}\|_{X}} \lesssim\|x\|_{X}
$$

holds for all $x \in X$.

Using the following notation, we can express the norms in a nicer way.

Notation 2.3. For any Hilbert space A, the term $A^{*}$ denotes its dual space equipped with the dual norm

$$
\|u\|_{A^{*}}:=\sup _{0 \neq w \in A} \frac{\langle u, w\rangle}{\|w\|_{A}},
$$

where $\langle u, \cdot\rangle:=u(\cdot)$ denotes the duality pairing.

For any Hilbert space $A$ and any scalar $\alpha>0$, the term $\alpha A$ denotes the space on the underlying set of the Hilbert space A equipped with the norm

$$
\|u\|_{\alpha A}^{2}:=\alpha\|u\|_{A}^{2} .
$$

For any two Hilbert spaces $A$ and $B$, the term $A \cap B$ denotes the space on the intersection of the underlying sets, $\{u \in A \cap B\}$, equipped with the norm

$$
\|u\|_{A \cap B}^{2}:=\|u\|_{A}^{2}+\|u\|_{B}^{2}
$$

and the term $A+B$ denotes the space on the algebraic sum of the underlying sets, $\left\{u_{1}+u_{2}: u_{1} \in A, u_{2} \in B\right\}$, equipped with the norm

$$
\|u\|_{A+B}^{2}:=\inf _{u_{1} \in A, u_{2} \in B, u=u_{1}+u_{2}}\left\|u_{1}\right\|_{A}^{2}+\left\|u_{2}\right\|_{B}^{2} .
$$


The spaces $A^{*}, \alpha A, A \cap B$ and $A+B$ are Hilbert spaces. The fact that $A^{*}$ is a Hilbert space follows directly from the Riesz representation theorem, see, e.g., Theorem 1.2 in [1]. The fact that $\alpha A$ is a Hilbert space is obvious and for the latter two see, e.g., Lemma 2.3.1 in [3].

We immediately see that the norm on $U$ can be rewritten as follows:

$$
\|u\|_{U}=\|u\|_{H^{1}(\Omega) \cap \beta L^{2}(\Omega)} .
$$

To reformulate the norm on $P$, we need the following regularity assumption.

(R) Regularity of the Stokes problem. Let $f \in\left[L^{2}(\Omega)\right]^{2}$ and $g \in H_{0}^{1}(\Omega) \cap L_{0}^{2}(\Omega)$ be arbitrary but fixed and $(u, p) \in\left[H_{0}^{1}(\Omega)\right]^{2} \times L_{0}^{2}(\Omega)$ be the solution of the Stokes problem, i.e., such that

$$
\begin{array}{ll}
(\nabla u, \nabla \tilde{u})_{L^{2}(\Omega)} & +\quad(p, \nabla \tilde{u})_{L^{2}(\Omega)} \\
(\nabla u, \tilde{p})_{L^{2}(\Omega)} & (f, \tilde{u})_{L^{2}(\Omega)} \\
& =(g, \tilde{p})_{L^{2}(\Omega)}
\end{array}
$$

holds for all $(\tilde{u}, \tilde{p}) \in\left[H_{0}^{1}(\Omega)\right]^{2} \times L_{0}^{2}(\Omega)$. Then $(u, p) \in\left[H^{2}(\Omega)\right]^{2} \times H^{1}(\Omega)$ and

$$
\|u\|_{H^{2}(\Omega)}^{2}+\|p\|_{H^{1}(\Omega)}^{2} \lesssim\|f\|_{L^{2}(\Omega)}^{2}+\|g\|_{H^{1}(\Omega)}^{2} .
$$

Lemma 2.4. The regularity assumption $(\mathbf{R})$ holds for $\Omega \subset \mathbb{R}^{2}$ being a convex polygonal domain.

Proof. Theorem 2 in [10] states (in the notation of the present paper) that provided $f \in\left[L^{2}(\Omega)\right]^{2}, g \in H^{1}(\Omega) \cap L_{0}^{2}(\Omega)$ and $\delta^{-1} g \in L^{2}(\Omega)$ that

$$
\|u\|_{H^{2}(\Omega)}^{2}+\|\nabla p\|_{L^{2}(\Omega)}^{2} \lesssim\|f\|_{L^{2}(\Omega)}^{2}+\|\nabla g\|_{L^{2}(\Omega)}^{2}+\left\|\delta^{-1} g\right\|_{L^{2}(\Omega)}^{2},
$$

is satisfied, where $\delta: \Omega \rightarrow \mathbb{R}$ is the distance to the closest corner of the polygonal domain $\Omega$. Lemma 2 in [10] states that $\left\|\delta^{-1} g\right\|_{L^{2}(\Omega)} \lesssim\|g\|_{H^{1}(\Omega)}$ is satisfied for all $g \in H_{0}^{1}(\Omega)$. Combining these results, we obtain that

$$
\|u\|_{H^{2}(\Omega)}^{2}+\|\nabla p\|_{L^{2}(\Omega)}^{2} \lesssim\|f\|_{L^{2}(\Omega)}^{2}+\|g\|_{H^{1}(\Omega)}^{2}
$$

is satisfied for all $f \in\left[L^{2}(\Omega)\right]^{2}$ and $g \in H_{0}^{1}(\Omega) \cap L_{0}^{2}(\Omega)$. As $p \in L_{0}^{2}(\Omega)$ was assumed, Poincaré's inequality states that $\|p\|_{H^{1}(\Omega)} \lesssim\|\nabla p\|_{L^{2}(\Omega)}$, which finishes the proof. $\square$

Note that we assume that $g$ satisfies homogeneous Dirichlet boundary conditions. This condition can be weakened but it is not possible to drop such a condition completely, cf. [10].

Lemma 2.5. On domains $\Omega$, where $(\mathbf{R})$ is satisfied,

$$
\|p\|_{P} \approx\|p\|_{L^{2}(\Omega)+\beta^{-1} H^{1}(\Omega)}
$$

holds for all $p \in L_{0}^{2}(\Omega)$.

For a proof of this lemma, see Theorem 3.2 in [16] (this proof only needs (R) for $f \in\left[L^{2}(\Omega)\right]^{2}$ and $\left.g=0\right)$.

The discretization of problem 2.1 is done using standard finite element techniques. We assume to have for $k=0,1,2, \ldots$ a sequence of grids obtained by uniform refinement. The grid size on grid level $k$ (length of the largest edge) is denoted by $h_{k}$. On each grid level $k$, we discretize the problem using the Galerkin approach, i.e., we have finite dimensional spaces $X_{k} \subset X$ and consider the following problem. Find $x_{k} \in X_{k}$ such that

$$
\mathcal{B}\left(x_{k}, \tilde{x}_{k}\right)=\mathcal{F}\left(\tilde{x}_{k}\right) \quad \text { for all } \tilde{x}_{k} \in X_{k} \text {. }
$$


Following the concept of mixed finite elements, we choose $X_{k}:=U_{k} \times P_{k}$, where $U_{k} \subset U$ and $P_{k} \subset P$ are obtained by a proper discretization, see below. Using a nodal basis, we can represent this problem in matrix-vector notation as follows:

$$
\mathcal{A}_{k} \underline{x}_{k}=\underline{f}_{k},
$$

where

$$
\mathcal{A}_{k}=\left(\begin{array}{cc}
A_{k} & B_{k}^{T} \\
B_{k} & 0
\end{array}\right), \quad \underline{x}_{k}=\left(\begin{array}{c}
\underline{u}_{k} \\
\underline{p}_{k}
\end{array}\right), \quad \underline{f}_{k}=\left(\begin{array}{c}
\underline{f}_{k} \\
\underline{g}_{k}
\end{array}\right)
$$

and the matrices $A_{k}$ and $B_{k}$ represent the scalar products $a(u, \tilde{u}):=(\nabla u, \nabla \tilde{u})_{L^{2}(\Omega)}+$ $\beta(u, \tilde{u})_{L^{2}(\Omega)}$ and $b(u, \tilde{p}):=(\nabla \cdot u, \tilde{p})_{L^{2}(\Omega)}$, respectively.

Here and in what follows, any underlined quantity, like $\underline{x}_{k}$, is the representation of the corresponding non-underlined quantity, here $x_{k}$, with respect to a nodal basis of the corresponding Hilbert space, here $X_{k}$.

The next step is to show the discrete stability condition, i.e., that

$$
\left\|x_{k}\right\|_{X} \lesssim \sup _{0 \neq \tilde{x}_{k} \in X_{k}} \frac{\mathcal{B}\left(x_{k}, \tilde{x}_{k}\right)}{\left\|\tilde{x}_{k}\right\|_{X}} \lesssim\left\|x_{k}\right\|_{X}
$$

holds for all $x_{k} \in X_{k}$.

To guarantee the discrete stability condition, we have to choose a discretization which is stable for the standard Stokes problem, particularly a discretization that satisfies the weak inf-sup condition, i.e.,

$$
\sup _{0 \neq u_{k} \in U_{k}} \frac{\left(\nabla \cdot u_{k}, p_{k}\right)_{L^{2}(\Omega)}}{\left\|u_{k}\right\|_{L^{2}(\Omega)}} \gtrsim\left\|\nabla p_{k}\right\|_{L^{2}(\Omega)}
$$

should hold for all $p_{k} \in P_{k}$. Note that this is a standard condition which guarantees that the chosen discretization is stable for the Stokes problem. In [2, 22] it was shown that condition $(2.5)$ is satisfied for the Taylor-Hood element $(P 1-P 2$-element) for polygonal domains where at least one vertex of each element is located in the interior of the domain. Here and in what follows we assume that the problem is discretized with the Taylor-Hood element and that the mesh satisfies the named condition. Using the weak-inf sup condition 2.5 we can show the following lemma.

Lemma 2.6. If the problem is discretized using the Taylor-Hood element, condition (A1a) is satisfied.

Proof. The estimate (2.16) in [15] states that

$$
\left\|x_{k}\right\|_{X_{1, k}} \lesssim \sup _{0 \neq \tilde{x}_{k} \in X_{k}} \frac{\mathcal{B}\left(x_{k}, \tilde{x}_{k}\right)}{\left\|\tilde{x}_{k}\right\|_{X_{1, k}}}
$$

holds for all $x_{k} \in X_{k}$, where

$$
\begin{aligned}
\left\|x_{k}\right\|_{X_{1, k}}^{2} & :=\left\|\left(u_{k}, p_{k}\right)\right\|_{X_{1, k}}^{2}:=\left\|u_{k}\right\|_{U_{1, k}}^{2}+\left\|p_{k}\right\|_{P_{1, k}}^{2}, \\
\left\|u_{k}\right\|_{U_{1, k}}^{2} & :=\left\|u_{k}\right\|_{H^{1}(\Omega)}^{2}+\beta\left\|u_{k}\right\|_{L^{2}(\Omega)}^{2} \text { and } \\
\left\|p_{k}\right\|_{P_{1, k}}^{2} & :=\sup _{0 \neq w_{k} \in U_{k}} \frac{\left(p_{k}, \nabla \cdot w_{k}\right)_{L^{2}(\Omega)}^{2}}{\left\|w_{k}\right\|_{H^{1}(\Omega)}^{2}+\beta\left\|w_{k}\right\|_{L^{2}(\Omega)}^{2}} .
\end{aligned}
$$

Here, the underlying sets of the Hilbert spaces $X_{1, k}, U_{1, k}$ and $P_{1, k}$ coincide with those of $X_{k}, U_{k}$ and $P_{k}$, respectively. Observe $\|\cdot\|_{U_{1, k}}=\|\cdot\|_{U}$. Lemma 2.2 in [15] states $\|\cdot\|_{P_{1, k}} \bar{\sim}\|\cdot\|_{P}$. This shows (A1a) as boundedness follows directly from (A1). $\square$

Note that the grids are obtained by uniform refinement. So, the discrete subsets are nested, i.e., $U_{k} \subseteq U_{k+1}, P_{k} \subseteq P_{k+1}$ and, consequently, $X_{k} \subseteq X_{k+1}$. 
3. A coupled multigrid method. The problem (2.4) shall be solved using a multigrid method. Starting from an initial approximation $\underline{x}_{k}^{(0)}$, one iterate of the multigrid method is given by the following two steps:

- Smoothing procedure: Compute

$$
\underline{x}_{k}^{(0, m)}:=\underline{x}_{k}^{(0, m-1)}+\hat{\mathcal{A}}_{k}^{-1}\left(\underline{f}_{k}-\mathcal{A}_{k} \underline{x}_{k}^{(0, m-1)}\right) \quad \text { for } m=1, \ldots, \nu
$$

with $\underline{x}_{k}^{(0,0)}:=\underline{x}_{k}^{(0)}$. The choice of the smoother (or, in other words, of the preconditioning matrix $\hat{\mathcal{A}}_{k}^{-1}$ ) will be discussed below.

- Coarse-grid correction:

- Compute the defect $\underline{f}_{k}-\mathcal{A}_{k} \underline{x}_{k}^{(0, \nu)}$ and restrict it to grid level $k-1$ using an restriction matrix $I_{k}^{k-1}$ :

$$
\underline{r}_{k-1}^{(1)}:=I_{k}^{k-1}\left(\underline{f}_{k}-\mathcal{A}_{k} \underline{x}_{k}^{(0, \nu)}\right) .
$$

- Determine the update $\underline{z}_{k-1}^{(1)}$ by solving the coarse-grid problem

$$
\mathcal{A}_{k-1} \underline{z}_{k-1}^{(1)}=\underline{r}_{k-1}^{(1)}
$$

approximately.

- Prolongate $\underline{z}_{k-1}^{(1)}$ to the grid level $k$ using an prolongation matrix $I_{k-1}^{k}$ and add the result to the previous iterate:

$$
\underline{x}_{k}^{(1)}:=\underline{x}_{k}^{(0, \nu)}+I_{k-1}^{k} \underline{z}_{k-1}^{(1)} .
$$

As we have assumed to have nested spaces, the intergrid-transfer matrices $I_{k-1}^{k}$ and $I_{k}^{k-1}$ are chosen in a canonical way: $I_{k-1}^{k}$ is the canonical embedding and the restriction $I_{k}^{k-1}$ is its transposed, i.e., $I_{k}^{k-1}=\left(I_{k-1}^{k}\right)^{T}$.

If the problem on the coarser grid is solved exactly (two-grid method), the coarsegrid correction is given by

$$
\underline{x}_{k}^{(1)}:=\underline{x}_{k}^{(0, \nu)}+I_{k-1}^{k} \mathcal{A}_{k-1}^{-1} I_{k}^{k-1}\left(\underline{f}_{k}-\mathcal{A}_{k} \underline{x}_{k}^{(0, \nu)}\right) .
$$

In practice, the problem $(3.2$ is approximately solved by applying one step (V-cycle) or two steps (W-cycle) of the multigrid method, recursively. On grid level $k=0$, the problem 3.2 is solved exactly.

To construct a multigrid convergence result based on Hackbusch's splitting of the analysis into smoothing property and approximation property, we have to introduce an appropriate framework.

Convergence is shown in the following $L^{2}$-like norms $\|\cdot \mid\|_{k}$ :

$$
\left\|x_{k}\right\|_{k}^{2}:=\left\|\underline{x}_{k}\right\|_{\mathcal{L}_{k}}^{2}:=\left(\mathcal{L}_{k} \underline{x}_{k}, \underline{x}_{k}\right)_{\ell^{2}},
$$

where

$$
\mathcal{L}_{k}:=\left(\begin{array}{cc}
\left(h_{k}^{-2}+\beta\right) M_{U, k} & \\
& h_{k}^{-2}\left(\beta+h_{k}^{-2}\right)^{-1} M_{P, k}
\end{array}\right),
$$

the matrices $M_{U, k}$ and $M_{P, k}$ are mass-matrices, representing the $L^{2}$-inner product in $U_{k}$ and $P_{k}$, respectively, and $(\cdot, \cdot)_{\ell^{2}}$ is the Euclidean scalar product.

The smoothing property and the approximation property, which we will show below, read as follows. 
- Smoothing property:

$$
\sup _{\tilde{x}_{k} \in X_{k}} \frac{\mathcal{B}\left(x_{k}^{(0, \nu)}-x_{k}^{*}, \tilde{x}_{k}\right)}{\left\|\tilde{x}_{k}\right\|_{k}} \leq \eta(\nu)\left\|x_{k}^{(0)}-x_{k}^{*}\right\|_{k}
$$

holds for some function $\eta(\nu)$ with $\lim _{\nu \rightarrow \infty} \eta(\nu)=0$. Here and in what follows, $x_{k}^{*} \in X_{k}$ is the exact solution of the discretized problem (2.3).

- Approximation property:

$$
\left\|x_{k}^{(1)}-x_{k}^{*}\right\|_{k} \leq C_{A} \sup _{\tilde{x}_{k} \in X_{k}} \frac{\mathcal{B}\left(x_{k}^{(0, \nu)}-x_{k}^{*}, \tilde{x}_{k}\right)}{\left\|\tilde{x}_{k}\right\|_{k}}
$$

holds for some constant $C_{A}>0$.

If we combine both conditions, we obtain

$$
\left\|x_{k}^{(1)}-x_{k}^{*}\right\|_{k} \leq q(\nu)\left\|x_{k}^{(0)}-x_{k}^{*}\right\|_{k},
$$

where $q(\nu)=C_{A} \eta(\nu)$. For $\nu$ large enough, we obtain $q(\nu)<1$, i.e., the convergence of the two-grid method. The convergence of the $\mathrm{W}$-cycle multigrid method can be shown under mild assumptions, see e.g. [9].

REMARK 3.1. The norm $\|\cdot\|_{k}$ is the discrete analog of $X_{0, k}:=U_{0, k} \times P_{0, k}:=$ $U_{k} \times P_{k}$, equipped with norms

$$
\begin{aligned}
\left\|x_{k}\right\|_{X_{0, k}}^{2} & :=\left\|\left(u_{k}, p_{k}\right)\right\|_{X_{0, k}}^{2}:=\left\|u_{k}\right\|_{U_{0, k}}^{2}+\left\|p_{k}\right\|_{P_{0, k}}^{2}, \\
\left\|u_{k}\right\|_{U_{0, k}}^{2} & :=h_{k}^{-2}\left\|u_{k}\right\|_{L^{2}(\Omega)}^{2}+\beta\left\|u_{k}\right\|_{L^{2}(\Omega)}^{2} \text { and } \\
\left\|p_{k}\right\|_{P_{0, k}}^{2} & :=\sup _{0 \neq w_{k} \in L^{2}(\Omega)} \frac{h_{k}^{-2}\left(p_{k}, w_{k}\right)_{L^{2}(\Omega)}^{2}}{h_{k}^{-2}\left\|w_{k}\right\|_{L^{2}(\Omega)}^{2}+\beta\left\|w_{k}\right\|_{L^{2}(\Omega)}^{2}} \\
& =h_{k}^{-2}\left(h_{k}^{-2}+\beta\right)^{-1}\left\|p_{k}\right\|_{L^{2}(\Omega)}^{2} .
\end{aligned}
$$

Note that this norm is obtained from the norm $\|\cdot\|_{X}$ by "replacing all differentials by

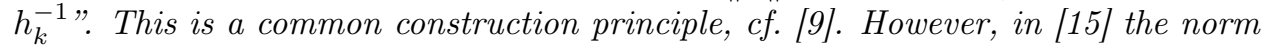

$$
\begin{aligned}
\left\|x_{k}\right\|_{\tilde{X}_{0, k}}^{2} & :=\left\|\left(u_{k}, p_{k}\right)\right\|_{\tilde{X}_{0, k}}^{2}:=\left\|u_{k}\right\|_{U_{0, k}}^{2}+\left\|p_{k}\right\|_{P_{1, k}}^{2}, \\
\left\|u_{k}\right\|_{U_{0, k}}^{2} & :=h_{k}^{-2}\left\|u_{k}\right\|_{L^{2}(\Omega)}^{2}+\beta\left\|u_{k}\right\|_{L^{2}(\Omega)}^{2} \text { and } \\
\left\|p_{k}\right\|_{P_{1, k}}^{2} & :=\sup _{0 \neq w_{k} \in U_{k}} \frac{\left(p_{k}, \nabla \cdot w_{k}\right)_{L^{2}(\Omega)}^{2}}{\left\|w_{k}\right\|_{H^{1}(\Omega)}^{2}+\beta\left\|w_{k}\right\|_{L^{2}(\Omega)}^{2}} .
\end{aligned}
$$

was used. Note that the norm for the velocity field coincides with our choice but the norm for the pressure distribution is still the original norm, as introduced in 2.6.).

4. Smoother and proof of the smoothing property. The choice of an appropriate smoother is a key issue in constructing a coupled multigrid method for an indefinite problem. In this paper, we introduce two kinds of smoothers. The first smoother is appropriate for a large class of problems including the model problem: the normal equation smoother, cf. [7], which reads as follows.

$$
\underline{x}_{k}^{(0, m)}:=\underline{x}_{k}^{(0, m-1)}+\tau \underbrace{\mathcal{L}_{k}^{-1} \mathcal{A}_{k} \mathcal{L}_{k}^{-1}}_{\hat{\mathcal{A}}_{k}^{-1}:=}\left(\underline{f}_{k}-\mathcal{A}_{k} \underline{x}_{k}^{(0, m-1)}\right) \quad \text { for } m=1, \ldots, \nu .
$$


Here, a fixed $\tau>0$ has to be chosen such that the spectral radius $\rho\left(\tau \hat{\mathcal{A}}_{k}^{-1} \mathcal{A}_{k}\right)$ is bounded away from 2 on all grid levels $k$ and for all choices of the parameter $\beta$.

REMARK 4.1. In Section 6.1 in [15] a normal equation smoother has been proposed. (There, this kind of smoothers was called distributive smoother.) Note that this class of smoothers depends on the choice of the Hilbert space norm. In the present paper, a scaled $L^{2}$-norm is used. Therefore, the Riesz isomorphism $\mathcal{L}_{k}$ can be easily inverted. However, in [15] a different norm was chosen, cf. Remark 3.1. For that norm, the realization of the inverse of the Riesz isomorphism involves the solution of one Poisson problem (for the pressure variable). So, two such problems have to be solved (approximately) in each smoothing step, cf. Section 8 in [15].

Using a standard inverse inequality, one can show that

$$
\left\|x_{k}\right\|_{X} \lesssim\left\|x_{k}\right\|_{k}
$$

is satisfied for all $x_{k} \in X_{k}$. Based on this result, using an eigenvalue analysis one can show the following lemma, cf. [7].

LEMMA 4.2. The damping parameter $\tau>0$ can be chosen independently of the grid level $k$ and the choice of the parameter $\beta$ such that

$$
\tau \rho\left(\hat{\mathcal{A}}_{k}^{-1} \mathcal{A}_{k}\right) \leq 2-\epsilon<2,
$$

holds for some constant $\epsilon>0$. For this choice of $\tau$, there is a constant $C_{S}>0$, independent of the grid level $k$ and the choice of the parameter $\beta$, such that the smoothing property 3.5 is satisfied with rate $\eta(\nu):=C_{S} \nu^{-1 / 2}$.

Certainly, the smoothing procedure (3.5) should be efficient-to-apply. Using the fact, that the mass matrices $M_{U, k}$ and $\bar{M}_{P, k}$ in 3.4 and their diagonals are spectrally equivalent under weak assumptions, for the practical realization of the normal equation smoother these mass matrices can be replaced by their diagonals.

The second smoother, which we propose, is a Uzawa type smoother, cf. [18. Here, one step of the smoother to compute $\underline{x}_{k}^{(0, m)}=\left(\underline{u}_{k}^{(0, m)}, \underline{p}_{k}^{(0, m)}\right)$ based on $\underline{x}_{k}^{(0, m-1)}=$ $\left(\underline{u}_{k}^{(0, m-1)}, \underline{p}_{k}^{(0, m-1)}\right)$ reads as follows:

$$
\begin{aligned}
\underline{u}_{k}^{(0, m-1 / 2)} & :=\underline{u}_{k}^{(0, m-1)}+\tau \hat{A}_{k}^{-1}\left(\underline{f}_{k}-A_{k} \underline{u}_{k}^{(0, m-1)}-B_{k}^{T} \underline{p}_{k}^{(0, m-1)}\right) \\
\underline{p}_{k}^{(0, m)} & :=\underline{p}_{k}^{(0, m-1)}-\sigma \hat{S}_{k}^{-1}\left(\underline{g}_{k}-B_{k} \underline{u}_{k}^{(0, m-1 / 2)}\right) \\
\underline{u}_{k}^{(0, m)} & :=\underline{u}_{k}^{(0, m-1)}+\tau \hat{A}_{k}^{-1}\left(\underline{f}_{k}-A_{k} \underline{u}_{k}^{(0, m-1)}-B_{k}^{T} \underline{p}_{k}^{(0, m)}\right),
\end{aligned}
$$

where $\hat{A}_{k}$ and $\hat{S}_{k}$ are the (1,1)-block and the $(2,2)$-block of $\mathcal{L}_{k}$, respectively. The damping parameters $\tau$ and $\sigma$ have to be chosen independently of the choice of $\beta$ and of the grid level $k$ such that 4.1 holds. The smoother can be rewritten in the compact notation (3.1, where

$$
\hat{\mathcal{A}}_{k}:=\left(\begin{array}{cc}
\tau^{-1} \hat{A}_{k} & B_{k}^{T} \\
B_{k} & \tau B_{k} \hat{A}_{k}^{-1} B^{T}-\sigma^{-1} \hat{S}_{k}
\end{array}\right) .
$$

The smoothing property can be shown using the theory introduced in [18.

LEMmA 4.3. Let $\hat{A}_{k}$ and $\hat{S}_{k}$ be the (1,1)-block and the (2,2)-block of $\mathcal{L}_{k}$, respectively. Then $\tau>0$ and $\sigma>0$ can be chosen independently of the grid level and the choice of the parameter $\beta$ such that

$$
\tau^{-1} \hat{A}_{k} \geq A_{k} \quad \text { and } \quad \sigma^{-1} \hat{S}_{k} \geq \tau B_{k} \hat{A}_{k}^{-1} B_{k}^{T}
$$


For this choice of $\tau$ and $\sigma$, there is a constant $C_{S}>0$, independent of the grid level $k$ and the choice of the parameter $\beta$, such that the smoothing property (3.5) is satisfied with rate $\eta(\nu):=C_{S} \nu^{-1 / 2}$.

Proof. The fact that $\tau>0$ and $\sigma>0$ can be chosen independently of the grid level and the choice of the parameter $\beta$ follows from standard inverse inequalities.

For the smoothing property, we apply Theorem 4 in 18 with the choice $\mathcal{K}_{k}:=$ $\mathcal{L}_{k}^{-1 / 2} \mathcal{A}_{k} \mathcal{L}_{k}^{-1 / 2}$ and $\hat{\mathcal{K}}_{k}:=\mathcal{L}_{k}^{-1 / 2} \hat{\mathcal{A}}_{k} \mathcal{L}_{k}^{-1 / 2}$. This immediately implies

$$
\left\|\mathcal{L}_{k}^{-1 / 2} \mathcal{A}_{k}\left(I-\hat{\mathcal{A}}_{k} \mathcal{A}_{k}\right)^{\nu} \mathcal{L}_{k}^{-1 / 2}\right\|_{\ell^{2}} \leq \eta(\nu)\left\|\mathcal{L}_{k}^{-1 / 2} \mathcal{A}_{k} \mathcal{L}_{k}^{-1 / 2}\right\|_{\ell^{2}}
$$

As $\underline{x}_{k}^{T} \mathcal{A}_{k} \underline{\tilde{x}}_{k}=\mathcal{B}\left(x_{k}, \tilde{x}_{k}\right) \lesssim\left\|x_{k}\right\|_{X}\left\|\tilde{x}_{k}\right\|_{X} \lesssim\left\|x_{k}\right\|\left\|_{k}\right\| \tilde{x}_{k}\left\|_{k}=\right\| \underline{x}_{k}\left\|_{\mathcal{L}_{k}}\right\| \underline{\tilde{x}}_{k} \|_{\mathcal{L}_{k}}$ holds for all $x_{k}, \tilde{x}_{k} \in X_{k}$, we obtain $\left\|\mathcal{L}_{k}^{-1 / 2} \mathcal{A}_{k} \mathcal{L}_{k}^{-1 / 2}\right\|_{\ell^{2}} \lesssim 1$, which finishes the proof.

REMARK 4.4. Uzawa type smoothers have also been considered in Section 6.2 in [15]. As for the case of normal equation smoothers (distributive smoothers) due to the particular norm that was chosen in [15], again a discrete Poisson problem for the pressure variable has to be solved in each smoothing step, cf. Section 8 in [15].

5. Proof of the approximation property. The proof of the approximation property (3.6) is done using the approximation theorem introduced in [20] which requires besides the conditions (A1) and (A1a) two more conditions (conditions (A3) and (A4)) involving, besides the Hilbert space $X$, two more Hilbert spaces $X_{-, k}:=$ $\left(X_{-},\|\cdot\|_{X_{-, k}}\right)$ and $X_{+, k}:=\left(X_{+},\|\cdot\|_{X_{+, k}}\right)$, which are chosen as follows.

As weaker space we choose $X_{-}:=U_{-} \times P_{-}$, where $U_{-}:=\left[L^{2}(\Omega)\right]^{2}$ and $P_{-}:=$ $\left[H_{0}^{1}(\Omega) \cap L_{0}^{2}(\Omega)\right]^{*}$. These Hilbert spaces are equipped with norms

$$
\begin{aligned}
\|x\|_{X_{-, k}}^{2} & :=\|(u, p)\|_{X_{-, k}}^{2}:=\|u\|_{U_{-, k}}^{2}+\|p\|_{P_{-, k}}^{2}, \\
\|u\|_{U_{-, k}}^{2} & :=h_{k}^{-2}\|u\|_{L^{2}(\Omega) \cap \beta\left[H_{0}^{1}(\Omega)\right]^{*}}^{2} \text { and } \\
\|p\|_{P_{-, k}}^{2} & :=h_{k}^{-2}\|p\|_{\left[H_{0}^{1}(\Omega)\right]^{*}+\beta^{-1} L_{0}^{2}(\Omega)}^{2} .
\end{aligned}
$$

REMARK 5.1. The idea behind the construction of the norm $\|\cdot\|_{X_{-, k}}$ is is to take the norm $\|\cdot\|_{X}$ and "replace all occurrences of $H^{1}$ by $h_{k}^{-1} L^{2}$ and all occurrences of $L^{2}$ by $h_{k}^{-1} H^{-1}$ ". This is different to the idea of constructing the norm $\|\cdot\|_{k}$, where "only the $H^{1}$-terms are replaced by $h_{k}^{-1} L^{2}$ and the $L^{2}$-terms are kept unchanged".

In the following, we show the approximation property in the norm $\|\cdot\|_{X_{-, k}}$, i.e.,

$$
\left\|x_{k}^{(1)}-x_{k}^{*}\right\|_{X_{-, k}} \leq \tilde{C}_{A} \sup _{\tilde{x}_{k} \in X_{k}} \frac{\mathcal{B}\left(x_{k}^{(0, \nu)}-x_{k}^{*}, \tilde{x}_{k}\right)}{\left\|\tilde{x}_{k}\right\|_{X_{-, k}}} .
$$

We will show below that this version of the approximation property is stronger than the required estimate (3.6), cf. Lemma 5.9. In classical approaches, cf. [20, 16], the approximation property is directly shown in the norm $\|\cdot \mid\|_{k}$.

Note that dual spaces are $\left(X_{-}\right)^{*}:=\left(U_{-}\right)^{*} \times\left(P_{-}\right)^{*}$, where $\left(U_{-}\right)^{*}=\left[L^{2}(\Omega)\right]^{2}$ and $\left(P_{-}\right)^{*}=H_{0}^{1}(\Omega) \cap L_{0}^{2}(\Omega)$, equipped with norms

$$
\begin{aligned}
\|\mathcal{F}\|_{\left(X_{-, k}\right)^{*}}^{2} & =\|(f, g)\|_{\left(X_{-, k}\right)^{*}}^{2}:=\|f\|_{\left(U_{-, k}\right)^{*}}^{2}+\|g\|_{\left(P_{-, k}\right)^{*}}^{2}, \\
\|f\|_{\left(U_{-, k}\right)^{*}}^{2} & =h_{k}^{2}\|f\|_{L^{2}(\Omega)+\beta^{-1} H_{0}^{1}(\Omega)}^{2} \text { and } \\
\|g\|_{\left(P_{-, k}\right)^{*}}^{2} & =h_{k}^{2}\|g\|_{H_{0}^{1}(\Omega) \cap \beta L_{0}^{2}(\Omega)}^{2} .
\end{aligned}
$$


As stronger space we choose $X_{+}:=U_{+} \times P_{+}$, where $U_{+}:=\left[H^{2}(\Omega) \cap H_{0}^{1}(\Omega)\right]^{2}$ and $P_{+}:=H^{1}(\Omega) \cap L_{0}^{2}(\Omega)$, equipped with norms

$$
\begin{aligned}
\|x\|_{X_{+, k}}^{2} & :=\|(u, p)\|_{X_{+, k}}^{2}:=\|u\|_{U_{+, k}}^{2}+\|p\|_{P_{+, k}}^{2}, \\
\|u\|_{U_{+, k}}^{2} & :=h_{k}^{2}\|u\|_{H^{2}(\Omega) \cap \beta H^{1}(\Omega)}^{2} \text { and } \\
\|p\|_{P_{+, k}}^{2} & :=h_{k}^{2}\|p\|_{H^{1}(\Omega)+\beta^{-1} H^{2}(\Omega)}^{2} .
\end{aligned}
$$

For showing the approximation property, we need some auxiliary results. The first result is a standard approximation error estimate:

THEOREM 5.2. On all grid levels $k$, the approximation error estimate

$$
\inf _{x_{k} \in X_{k}}\left\|x-x_{k}\right\|_{X} \lesssim\|x\|_{X_{+, k}} \quad \text { for all } x \in X_{+}
$$

is satisfied.

The proof of this theorem is rather easy, as it can be shown for $U$ and $P$ separately. For completeness, we give a proof of this theorem in the Appendix. The next step is to show a regularity result for the generalized Stokes problem:

(A4) For all grid levels $k$ and all $\mathcal{F} \in\left(X_{-}\right)^{*}$, the solution of the problem,

$$
\text { find } x_{\mathcal{F}} \in X \text { such that } \quad \mathcal{B}\left(x_{\mathcal{F}}, \tilde{x}\right)=\mathcal{F}(\tilde{x}) \quad \text { for all } \tilde{x} \in X \text {, }
$$

satisfies $x_{\mathcal{F}} \in X_{+}$and $\left\|x_{\mathcal{F}}\right\|_{X_{+, k}} \lesssim\|\mathcal{F}\|_{\left(X_{-, k}\right)^{*}}$.

For showing (A4), we need a standard regularity result for the Poisson problem.

(R1) Regularity of the Poisson problem. For all $g \in L^{2}(\Omega)$, the solution of the problem, find $p_{g} \in H^{1}(\Omega) \cap L_{0}^{2}(\Omega)$ such that

$$
\left(\nabla p_{g}, \nabla \tilde{p}\right)_{H^{1}(\Omega)}=(g, \tilde{p})_{L^{2}(\Omega)} \quad \text { for all } \tilde{p} \in H^{1}(\Omega) \cap L_{0}^{2}(\Omega),
$$

satisfies $p_{g} \in H^{2}(\Omega)$ and $\left\|p_{g}\right\|_{H^{2}(\Omega)} \lesssim\|g\|_{L^{2}(\Omega)}$.

Lemma 5.3. The regularity assumption (R1) holds for $\Omega \subset \mathbb{R}^{2}$ being a convex polygonal domain.

For a proof oft this lemma, see, e.g., [8]. The first step of the proof of (A4), is to show that $x_{\mathcal{F}}$ is in the desired space.

LEMma 5.4. Suppose that $\Omega$ is such that the regularity assumption $(\mathbf{R})$ holds. Let $\mathcal{F} \in\left(X_{-}\right)^{*}$ and let $x_{\mathcal{F}}$ be the solution of (5.2). Then $x_{\mathcal{F}} \in X_{+}$.

Proof. Let $\mathcal{F}(\tilde{u}, \tilde{p}):=(f, \tilde{u})_{L^{2}(\Omega)}+(g, \tilde{p})_{L^{2}(\Omega)}$ for $f \in\left[L^{2}(\Omega)\right]^{2}$ and $g \in H_{0}^{1}(\Omega) \cap$ $L_{0}^{2}(\Omega)$. Rewrite the problem as follows:

$$
\begin{aligned}
\left(\nabla u_{\mathcal{F}}, \nabla \tilde{u}\right)_{L^{2}(\Omega)} & +\quad\left(p_{\mathcal{F}}, \nabla \cdot \tilde{u}\right)_{L^{2}(\Omega)} \\
\left(\nabla \cdot u_{\mathcal{F}}, \tilde{p}\right)_{L^{2}(\Omega)} & =(f, \tilde{u})_{L^{2}(\Omega)}-\beta\left(u_{\mathcal{F}}, \tilde{u}\right)_{L^{2}(\Omega)} \\
& =(g, \tilde{p})_{L^{2}(\Omega)}
\end{aligned}
$$

for all $\tilde{u} \in U$ and $\tilde{p} \in P$. As $f-\beta u_{\mathcal{F}} \in\left[L^{2}(\Omega)\right]^{2}$ and $g \in H_{0}^{1}(\Omega) \cap L_{0}^{2}(\Omega)$, we obtain using regularity assumption $(\mathbf{R})$ immediately that $x_{\mathcal{F}} \in X_{+}$.

Note that the combination of the argument used in the proof of this lemma and condition (A1) implies $\left\|x_{\mathcal{F}}\right\|_{X_{+, k}} \leq C(\beta)\|\mathcal{F}\|_{\left(X_{-, k}\right)^{*}}$, where $C(\beta)$ is a constant depending on $\beta$. For showing a robust estimate, we need to do some more work.

LEMma 5.5. Suppose that $\Omega$ is such that the assumptions $(\mathbf{R})$ and $(\mathbf{R} 1)$ are satisfied. Let $\mathcal{F} \in\left(X_{-}\right)^{*}$ and let $x_{\mathcal{F}}=\left(u_{\mathcal{F}}, p_{\mathcal{F}}\right)$ be the solution of 5.2 . Then

$$
\left\|p_{\mathcal{F}}\right\|_{P_{+, k}}^{2} \lesssim\left\|u_{\mathcal{F}}\right\|_{U_{+, k}}^{2}+\|\mathcal{F}\|_{\left(X_{-, k}\right)^{*}}^{2}
$$


LEMmA 5.6. Suppose that $\Omega$ is such that the assumption $(\mathbf{R})$ is satisfied. Let $\mathcal{F} \in\left(X_{-}\right)^{*}$ and let $x_{\mathcal{F}}=\left(u_{\mathcal{F}}, p_{\mathcal{F}}\right)$ be the solution of (5.2). Then

$$
\left\|u_{\mathcal{F}}\right\|_{U_{+, k}}^{2} \lesssim\|\mathcal{F}\|_{\left(X_{-, k}\right) *}\left\|x_{\mathcal{F}}\right\|_{X_{+, k}} \text {. }
$$

The proofs of these two lemmas can be found in the Appendix. Using them, we can show (A4):

THEOREM 5.7. If $\Omega$ is such that the regularity assumptions $(\mathbf{R})$ and $(\mathbf{R} \mathbf{1})$ are satisfied, then the regularity statement (A4) holds.

Proof. Note that Lemma 5.4 already states that $x_{\mathcal{F}} \in X_{+}$. It remains to show $\left\|x_{\mathcal{F}}\right\|_{X_{+, k}} \lesssim\|\mathcal{F}\|_{\left(X_{-, k}\right)}$ Note that Lemma 5.5 implies immediately

$$
\left\|x_{\mathcal{F}}\right\|_{X_{+, k}}^{2}=\left\|u_{\mathcal{F}}\right\|_{U_{+, k}}^{2}+\left\|p_{\mathcal{F}}\right\|_{P_{+, k}}^{2} \lesssim\left\|u_{\mathcal{F}}\right\|_{U_{+, k}}^{2}+\|\mathcal{F}\|_{\left(X_{-, k}\right)^{*}}^{2}
$$

If we combine this result with the statement of Lemma 5.6 , we obtain

$$
\left\|x_{\mathcal{F}}\right\|_{X_{+, k}}^{2} \leq C\left(\left\|x_{\mathcal{F}}\right\|_{X_{+, k}}\|\mathcal{F}\|_{\left(X_{-, k}\right)^{*}}+\|\mathcal{F}\|_{\left(X_{-, k}\right)^{*}}^{2}\right)
$$

for some constant $C>0$ (independent of $k$ and $\beta$ ) which implies

$$
\left\|x_{\mathcal{F}}\right\|_{X_{+, k}} \leq \frac{1}{2}\left(C+\sqrt{4 C+C^{2}}\right)\|\mathcal{F}\|_{\left(X_{-, k}\right)^{*}}
$$

and the desired estimate. This finishes the proof. $\square$

As we have shown that the conditions (A1), (A1a), (A3) and (A4) are satisfied, we can apply Theorem 4.1 in [20] and obtain:

THEOREM 5.8. Assume that $\Omega$ is such that $(\mathbf{R})$ and $(\mathbf{R 1})$ are satisfied and assume that the problem is discretized using the Taylor-Hood element. Then the coarsegrid correction (3.3) satisfies the approximation property (5.1) where $\tilde{C}_{A}$ only depends on the constants that appear in the conditions (A1), (A1a), (A3) and (A4).

This shows (5.1), the approximation property in the norm $\|\cdot\|_{X_{-, k}}$, however we need $(3.6)$, the approximation property in the norm $\|\cdot\| \|_{k}$. The final step is to relate these two norms:

LEMMA 5.9. The estimate

$$
\left\|x_{k} \mid\right\|_{k} \lesssim\left\|x_{k}\right\|_{X_{-, k}}
$$

holds for all $x_{k} \in X_{k}$

Proof. First note that it suffices to show $\left\|u_{k}\right\|_{U, k} \lesssim\left\|u_{k}\right\|_{U_{-, k}}$ and $\left\|p_{k}\right\| \|_{P, k} \lesssim$ $\left\|p_{k}\right\|_{P_{-, k}}$. We obtain using the definition of $\|\cdot\|_{U_{-, k}}$, the choice $\tilde{u}:=u_{k} \in\left[H_{0}^{1}(\Omega)\right]^{2}$ and a standard inverse inequality

$$
\begin{aligned}
& \left\|u_{k}\right\|_{U_{-, k}}^{2}=\sup _{0 \neq \tilde{u} \in\left[L^{2}(\Omega)\right]^{2}} \frac{\left(u_{k}, \tilde{u}\right)_{L^{2}(\Omega)}^{2}}{h_{k}^{2}\|\tilde{u}\|_{L^{2}(\Omega)+\beta^{-1} H_{0}^{1}(\Omega)}^{2}} \gtrsim \frac{\left(u_{k}, u_{k}\right)_{L^{2}(\Omega)}^{2}}{h_{k}^{2}\left\|u_{k}\right\|_{L^{2}(\Omega)+\beta^{-1} H_{0}^{1}(\Omega)}^{2}} \\
& =\frac{\left(u_{k}, u_{k}\right)_{L^{2}(\Omega)}^{2}}{\inf _{w \in\left[H_{0}^{1}(\Omega)\right]^{2}} h_{k}^{2}\left\|w-u_{k}\right\|_{L^{2}(\Omega)}^{2}+h_{k}^{2}\|w\|_{\beta^{-1} H^{1}(\Omega)}^{2}} \\
& \gtrsim \frac{\left(u_{k}, u_{k}\right)_{L^{2}(\Omega)}^{2}}{\inf _{w_{k} \in U_{k}} h_{k}^{2}\left\|w_{k}-u_{k}\right\|_{L^{2}(\Omega)}^{2}+h_{k}^{2}\left\|w_{k}\right\|_{\beta^{-1} H^{1}(\Omega)}^{2}} \\
& \gtrsim \frac{\left(u_{k}, u_{k}\right)_{L^{2}(\Omega)}^{2}}{\inf _{w_{k} \in U_{k}} h_{k}^{2}\left\|w_{k}-u_{k}\right\|_{L^{2}(\Omega)}^{2}+\left\|w_{k}\right\|_{\beta^{-1} L^{2}(\Omega)}^{2}}
\end{aligned}
$$


For the proof of the inequality for the pressure variable, we define on each grid level a function $\psi_{k}: \Omega \rightarrow \mathbb{R}$ as follows. The function $\psi_{k}$ is 0 on all vertices which are located on $\partial \Omega, 1$ on all other vertices (interior vertices) and linear within each element (Courant element).

In what follows, $\hat{P}_{k}$ is the space of functions obtained by discretizing the space $H^{1}(\Omega)$ using the standard Courant element. Note that - as we have used the Taylor Hood element - the identity $\hat{P}_{k}=\left\{p_{k}+a: p_{k} \in P_{k}\right.$ and $\left.a \in \mathbb{R}\right\}$ holds.

Note that we obtain using the definition of $\|\cdot\|_{P_{-, k}}$ and $q \subseteq L_{0}^{2}(\Omega)$ that

$$
\left\|p_{k}\right\|_{P_{-, k}}^{2}=\sup _{0 \neq q \in H_{0}^{1}(\Omega) \cap L_{0}^{2}(\Omega)} \frac{\left(p_{k}, q\right)_{L^{2}(\Omega)}^{2}}{h_{k}^{2}\|q\|_{H_{0}^{1}(\Omega) \cap \beta L^{2}(\Omega)}^{2}}=\sup _{0 \neq q \in H_{0}^{1}(\Omega) \cap L_{0}^{2}(\Omega)} \frac{\left(p_{k}-a, q\right)_{L^{2}(\Omega)}^{2}}{h_{k}^{2}\|q\|_{H_{0}^{1}(\Omega) \cap \beta L^{2}(\Omega)}^{2}}
$$

is satisfied, where $a \in \mathbb{R}$ is chosen such that $\left(p_{k}-a\right) \psi_{k} \in L_{0}^{2}(\Omega)$. Observe that $q:=\left(p_{k}-a\right) \psi_{k} \in H_{0}^{1}(\Omega) \cap L_{0}^{2}(\Omega)$. Using this choice, we obtain

$$
\left\|p_{k}\right\|_{P_{-, k}}^{2} \geq \frac{\left(p_{k}-a,\left(p_{k}-a\right) \psi_{k}\right)_{L^{2}(\Omega)}^{2}}{h_{k}^{2}\left\|\left(p_{k}-a\right) \psi_{k}\right\|_{H^{1}(\Omega)}^{2}+\beta h_{k}^{2}\left\|\left(p_{k}-a\right) \psi_{k}\right\|_{L^{2}(\Omega)}^{2}} .
$$

Using $\left|\psi_{k}\right| \leq 1$, we obtain $\left\|\left(p_{k}-a\right) \psi_{k}\right\|_{L^{2}(\Omega)}^{2} \leq\left\|p_{k}-a\right\|_{L^{2}(\Omega)}^{2}$. The following two inequalities hold:

$$
\begin{aligned}
\left\|\psi_{k} p_{k}\right\|_{H^{1}(\Omega)}^{2} & \lesssim h_{k}^{-2}\left\|p_{k}\right\|_{L^{2}(\Omega)}^{2} \text { and } \\
\left(p_{k}, \psi_{k} p_{k}\right)_{L^{2}(\Omega)} & \gtrsim\left(p_{k}, p_{k}\right)_{L^{2}(\Omega)} .
\end{aligned}
$$

Proofs are given in the Appendix. Using these inequalities and $p_{k} \in L_{0}^{2}(\Omega)$, we obtain

$$
\begin{aligned}
\left\|p_{k}\right\|_{P_{-, k}}^{2} & \gtrsim \frac{\left(p_{k}-a, p_{k}-a\right)_{L^{2}(\Omega)}^{2}}{\left\|p_{k}-a\right\|_{L^{2}(\Omega)}^{2}+\beta h_{k}^{2}\left\|p_{k}-a\right\|_{L^{2}(\Omega)}^{2}} \\
& =\left(1+\beta h_{k}^{2}\right)^{-1}\left\|p_{k}-a\right\|_{L^{2}(\Omega)}^{2} \geq\left(1+\beta h_{k}^{2}\right)^{-1}\left\|p_{k}\right\|_{L^{2}(\Omega)}^{2}=\left\|p_{k}\right\|_{P, k}^{2},
\end{aligned}
$$

which finishes the proof. $\square$

Using this lemma and the approximation property (5.1), we obtain the approximation property 3.6 . Together with the smoothing property, we obtain:

ThEOREM 5.10. Assume that

- $\Omega$ is such that the regularity assumptions (R) and (R1) are satisfied,

- the problem is discretized using the Taylor-Hood element and

- one of the smoothers proposed in this paper is used.

Then

$$
\left\|x_{k}^{(1)}-x_{k}^{*}\right\|_{k} \leq q(\nu)\left\|x_{k}^{(0)}-x_{k}^{*}\right\|_{k}
$$

holds with $q(\nu):=C_{S} C_{A} \nu^{-1 / 2}$, where the constants $C_{A}$ and $C_{S}$ are independent of the grid level $k$ and the choice of the parameter $\beta$. For $\nu>\left(C_{S} C_{A}\right)^{2}$, the two-grid method converges with convergence rate $q(\nu)<1$.

The convergence of the $\mathrm{W}$-cycle multigrid follows under weak assumptions, cf. [9].

6. Numerical Results. In this section, we illustrate the convergence theory presented within this paper with numerical results. For the numerical experiments, the domain $\Omega$ was chosen to be the unit square $\Omega:=(0,1)^{2}$. As mentioned in Section 2 , the weak inf-sup-condition 2.5 can be shown for the Taylor-Hood element only if at least 
one vertex of each element is in the interior of the domain $\Omega$. As this is not satisfied for the the standard decomposition of the unit square into two triangular elements, we choose the coarsest grid level $k=0$ to be a decomposition of the domain $\Omega$ into 8 triangles, as seen in Figure 6.1. The grid levels $k=1,2, \ldots$ were constructed by uniform refinement, i.e., every triangle was decomposed into four subtriangles.
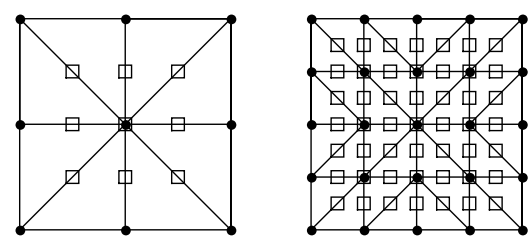

Figure 6.1. Discretization on grid levels $k=1$ and $k=2$, where the squares denote the degrees of freedom of (the components of) the velocity field $u$ and the dots denote the degrees of freedom of pressure distribution $p$

The right-hand-side functions $f$ and $g$ have been chosen such that the solution of the problem on grid level $k$ is the $L^{2}$-projection of the exact solution

$$
u\left(\xi_{1}, \xi_{2}\right):=\phi\left(\xi_{1}, \xi_{2}\right)\left(\begin{array}{c}
\xi_{2}-\frac{1}{2} \\
\frac{1}{2}-\xi_{1}
\end{array}\right) \quad \text { and } \quad p\left(\xi_{1}, \xi_{2}\right):=\phi\left(\xi_{1}, \xi_{2}\right),
$$

into $X_{k}$, where $\phi\left(\xi_{1}, \xi_{2}\right):=\max \left\{0, \min \left\{1,2-4 \sqrt{\left(\xi_{1}-\frac{1}{2}\right)^{2}+\left(\xi_{2}-\frac{1}{2}\right)^{2}}\right\}\right\}$.

For solving the discretized problem, we have used the proposed W-cycle multigrid method. As mentioned above, the matrix $\mathcal{L}_{k}$, introduced in Section 3 can be replaced by any spectrally equivalent matrix. As $\mathcal{L}_{k}$ should be easy-to-invert, $\mathcal{L}_{k}$ should be a diagonal matrix. The convergence theory indicates how $\mathcal{L}_{k}$ should be scaled, however only up to constants. Numerical experiments indicated that it is reasonable to choose

$$
\mathcal{L}_{k}:=\left(\begin{array}{cc}
\hat{A}_{k} & \\
& \hat{S}_{k}
\end{array}\right), \quad \text { where } \quad \hat{A}_{k}:=\operatorname{diag} A_{k} \quad \text { and } \quad \hat{S}_{k}:=\operatorname{diag} B_{k} \hat{A}_{k}^{-1} B_{k}^{T},
$$

i.e., to choose these constants as they appear naturally in the diagonal of $A_{k}$, the $(1,1)$ block of $\mathcal{A}_{k}$, and in the diagonal of the inexact Schur complement. As this choice of $\mathcal{L}_{k}$ is spectrally equivalent to the matrix $\mathcal{L}_{k}$, introduced in Section 3 , this choice is still covered by the convergence theory. For this choice, the damping parameter was chosen to be $\tau=0.35$, for all grid levels $k$ and all choices of $\beta$.

For the Uzawa type smoother, the matrices $\hat{A}_{k}$ and $\hat{S}_{k}$ have been chosen as introduced in (6.1) and the damping parameters have been chosen to be $\tau=\sigma=0.8$ for all grid levels $k$ and all choices of $\beta$. Again, the choice of this section is covered by the convergence theory.

The number of iterations and the convergence rate were measured as follows: we start with $\underline{x}_{k}^{(0)}$ and measure the reduction of the error in each step using the norm $\|\mid \cdot\|_{k}$. The iteration was stopped when the initial error was reduced by a factor of $\epsilon=10^{-9}$. The convergence rate $q$ is the mean convergence rate in this iteration, i.e.,

$$
q=\left(\frac{\left\|x_{k}^{(n)}-x_{k}^{*}\right\|_{k}}{\left\|x_{k}^{(0)}-x_{k}^{*}\right\|_{k}}\right)^{1 / n},
$$

where $n$ is the number of iterations needed to reach the stopping criterion. Here, $x_{k}^{*}$ is the exact solution and $x_{k}^{(i)}$ is the $i$-th iterate. 


\begin{tabular}{lllllllll}
\hline$\nu=1+1$ & $\nu=2+2$ & $\nu=3+3$ & $\nu=4+4$ & $\nu=8+8$ & & $\nu=16+16$ \\
\hline$n$ & $q$ & $n$ & $q$ & $n$ & $q$ & $n$ & $q$ & $n$
\end{tabular}

Normal equation smoother

\begin{tabular}{|c|c|c|c|c|c|c|c|c|c|c|c|c|c|c|}
\hline 88 & 0.789 & 46 & 0.634 & 30 & 0.496 & & 24 & \multicolumn{2}{|c|}{0.412} & 17 & \multicolumn{2}{|c|}{0.293} & 11 & 0.148 \\
\hline \multicolumn{15}{|c|}{ Uzawa type smoother } \\
\hline \multicolumn{2}{|c|}{ divergent } & \multicolumn{2}{|c|}{ divergent } & 14 & \multicolumn{2}{|c|}{0.212} & 20 & \multicolumn{2}{|c|}{0.347} & 6 & \multicolumn{2}{|c|}{0.031} & 5 & 0.008 \\
\hline \multicolumn{15}{|c|}{ Asymptotic decay of the convergence rate predicted by theory $\left(\nu^{-1 / 2}\right)$} \\
\hline \multicolumn{3}{|c|}{0.707} & 0.500 & & \multicolumn{3}{|c|}{0.408} & \multicolumn{3}{|c|}{0.353} & \multicolumn{2}{|c|}{0.250} & \multicolumn{2}{|r|}{0.177} \\
\hline \multicolumn{15}{|c|}{$\begin{array}{c}\text { TABLE } 6.1 \\
\text { Number of iterations } n \text { and mean convergence rate } q \text { for the normal equation smoother and th } \\
\text { Uzawa type smoother depending with } \nu=\nu_{\text {pre }}+\nu_{\text {post }} \text { smoothing steps on grid level } k=4 \text { for } \beta=1\end{array}$} \\
\hline \multicolumn{3}{|c|}{$\beta=0$} & \multicolumn{2}{|c|}{$\beta=10^{2}$} & \multicolumn{3}{|c|}{$\beta=10^{4}$} & \multicolumn{3}{|c|}{$\beta=10^{6}$} & \multicolumn{2}{|c|}{$\beta=10^{8}$} & \multicolumn{2}{|c|}{$\beta=10^{10}$} \\
\hline & $n$ & $q$ & $n$ & $q$ & & $q$ & & & $q$ & & & $q$ & $n$ & $q$ \\
\hline$k=4$ & 30 & 0.496 & 30 & 0.493 & 30 & & 96 & 68 & 0.73 & & 71 & 0.745 & 71 & 0.745 \\
\hline$k=5$ & 29 & 0.489 & 29 & 0.488 & 22 & 0. & & 64 & 0.72 & & 70 & 0.744 & 71 & 0.745 \\
\hline$k=6$ & 29 & 0.484 & 29 & 0.486 & 27 & 0. & & 52 & 0.67 & & 70 & 0.743 & 71 & 0.745 \\
\hline$k=7$ & 28 & 0.475 & 28 & 0.475 & 28 & & & 35 & 0.55 & & 70 & 0.742 & 71 & 0.745 \\
\hline$k=8$ & 28 & 0.469 & 28 & 0.469 & 28 & & & 20 & 0.34 & & 67 & 0.732 & 71 & 0.746 \\
\hline
\end{tabular}

TABLE 6.2

Number of iterations $n$ and mean convergence rate $q$ for the normal equation smoother with $\nu=3+3$ smoothing steps

In Table 6.1 we compare for a fixed grid level (level $k=4$ ) and the fixed choice $\beta=1$ the convergence rates for several choices of $\nu$, the number of pre- and postsmoothing steps. We see that the convergence rate behaves approximately like $\nu^{-1 / 2}$, if the number of smoothing steps is increased. This is consistent with the theory which guarantees the convergence rate being bounded by $C \nu^{-1 / 2}$. We observe that the preconditioned normal equation smoother already converges for $\nu=1+1$ smoothing steps, while for the Uzawa type smoother $\nu=3+3$ smoothing steps are necessary.

In Tables 6.2 and 6.3 we compare various grid levels $k$ and choices of the parameter $\beta$. We have used a fixed choice of $\nu=3+3$ smoothing steps. First we observe that, for both smoothers, the number of iterations seems to be well-bounded for all grid levels $k$ which yields an optimal convergence behavior. Moreover, we see that the number of iterations is also well-bounded for a wide range of choices of the parameter $\beta$, i.e., we observe also robust convergence as predicted by the convergence theory. Comparing both kinds of smoothers, we see that the Uzawa type smoother leads to much faster convergence rates than the preconditioned normal equation smoother. Note that moreover the computational complexity of the Uzawa type smoother (per iteration) is slightly smaller than the complexity of the normal equation smoother.

It has to be mentioned that for the model problem, also the V-cycle multigrid method converges with rates comparable to the convergence rates of the $\mathrm{W}$-cycle multigrid method. However, the V-cycle is not covered by the convergence theory. 


\begin{tabular}{lllllllllllllll}
\hline & \multicolumn{3}{c}{$\beta=0$} & \multicolumn{3}{c}{$\beta=10^{2}$} & \multicolumn{3}{c}{$\beta=10^{4}$} & \multicolumn{3}{c}{$\beta=10^{6}$} & \multicolumn{3}{c}{$\beta=10^{8}$} & \multicolumn{2}{c}{$\beta=10^{10}$} \\
& $n$ & $q$ & $n$ & $q$ & $n$ & $q$ & $n$ & $q$ & $n$ & $q$ & $n$ & $q$ \\
\hline$k=4$ & 14 & 0.212 & 13 & 0.201 & 6 & 0.030 & 7 & 0.051 & 8 & 0.059 & 8 & 0.059 \\
$k=5$ & 13 & 0.194 & 13 & 0.193 & 9 & 0.095 & 7 & 0.043 & 7 & 0.050 & 7 & 0.050 \\
$k=6$ & 12 & 0.176 & 12 & 0.176 & 11 & 0.145 & 6 & 0.024 & 7 & 0.047 & 7 & 0.048 \\
$k=7$ & 12 & 0.166 & 12 & 0.166 & 11 & 0.150 & 5 & 0.013 & 7 & 0.044 & 7 & 0.045 \\
$k=8$ & 11 & 0.147 & 11 & 0.147 & 11 & 0.145 & 8 & 0.058 & 7 & 0.038 & 7 & 0.043 \\
\hline
\end{tabular}

TABLE 6.3

Number of iterations $n$ and mean convergence rate $q$ for the Uzawa type smoother with $\nu=3+3$ smoothing steps

The numerical experiments done by the author have shown that the convergence rates can be improved slightly by adjusting the choice of the parameters to the grid levels and the choice of $\beta$. However, the main goal of this paper is to show that the proposed method also works well for fixed choices of the parameter.

7. Conclusions and Further Work. In the present paper we have proposed a coupled multigrid solver for the generalized Stokes problem where the smoothing property is shown in the scaled $L^{2}$-norm \|\|$\cdot \|_{k}$. This allows to construct a multigrid method where the smoother is a simple linear iteration (which consists only of divisions and the multiplication of vectors with the system matrix $\mathcal{A}_{k}$ ). In the present paper, a preconditioned normal equation smoother and an Uzawa type smoother have been chosen but it seems possible to find also other smoothers which satisfy the smoothing property in the norm $\|\cdot \cdot\|_{k}$.

The convergence rates observed for the multigrid method proposed in the present paper are comparable with the rates observed for the methods proposed in [15]. Note that for applying the methods proposed in the named paper, it is necessary to solve a Poisson problem in each smoothing step. This is not needed for the method proposed in the present paper. The main contribution of this paper is a new way of setting up the norms where the convergence is sown in. The technique of the convergence proof that has been applied in the present paper is also extendable to the Stokes control problem, cf. [19.

Acknowledgments. The author thanks Markus Kollmann for providing code used to compute the numerical results presented in this paper. Moreover, the support of the Austrian Science Funds (FWF), the University of Oxford and the Technische Universität Chemnitz are gratefully acknowledged. The author thanks the referee for many helpful suggestions.

8. Appendix. Proof of Theorem 5.2. Note that it suffices to show approximation error results for the individual variables separately. Using a standard interpolation operator $\Pi_{k}:\left[H^{2}(\Omega)\right]^{2} \rightarrow U_{k}$, we obtain

$$
\left\|u-\Pi_{k} u\right\|_{L^{2}(\Omega)}^{2} \lesssim h_{k}^{2}\|u\|_{H^{1}(\Omega)}^{2} \quad \text { and } \quad\left\|u-\Pi_{k} u\right\|_{H^{1}(\Omega)}^{2} \lesssim h_{k}^{2}\|u\|_{H^{2}(\Omega)}^{2}
$$

for all $u \in\left[H^{2}(\Omega)\right]^{2}$ and therefore

$$
\begin{aligned}
& \inf _{u_{k} \in U_{k}}\left\|u-u_{k}\right\|_{U}^{2} \leq\left\|u-\Pi_{k} u\right\|_{U}^{2}=\left\|u-\Pi_{k} u\right\|_{H^{1}(\Omega)}^{2}+\beta\left\|u-\Pi_{k} u\right\|_{L^{2}(\Omega)}^{2} \\
& \quad \lesssim h_{k}^{2}\left(\|u\|_{H^{2}(\Omega)}^{2}+\beta\|u\|_{H^{1}(\Omega)}^{2}\right)=\|u\|_{U_{+, k}}^{2} .
\end{aligned}
$$


A similar argument is possible for the pressure distribution. The standard approximation error estimates

$$
\inf _{p_{k} \in P_{k}}\left\|p-p_{k}\right\|_{L^{2}(\Omega)}^{2} \lesssim h_{k}^{2}\|p\|_{H^{1}(\Omega)}^{2} \quad \text { and } \quad \inf _{p_{k} \in P_{k}}\left\|p-p_{k}\right\|_{H^{1}(\Omega)}^{2} \lesssim h_{k}^{2}\|p\|_{H^{2}(\Omega)}^{2}
$$

hold for all $p \in H^{1}(\Omega)$ or $p \in H^{2}(\Omega)$, respectively. They imply

$$
\begin{aligned}
& \inf _{p_{k} \in P_{k}}\left\|p-p_{k}\right\|_{P}^{2}=\inf _{\substack{p_{1} \in L^{2}(\Omega) \\
p_{2} \in H^{1}(\Omega) \\
p_{1}+p_{2}=p}} \inf _{\substack{1, k \\
\text { in }}}\left\|p_{k}-p_{1, k}\right\|_{L^{2}(\Omega)}^{2}+\inf _{p_{2, k} \in P_{k}}\left\|p_{2}-p_{2, k}\right\|_{\beta^{-1} H^{1}(\Omega)}^{2} \\
& \leq \inf _{p_{1} \in H^{1}(\Omega)} \inf _{p_{1, k} \in P_{k}}\left\|p_{1}-p_{1, k}\right\|_{L^{2}(\Omega)}^{2}+\inf _{p_{2, k} \in P_{k}}\left\|p_{2}-p_{2, k}\right\|_{\beta^{-1} H^{1}(\Omega)}^{2} \\
& p_{2} \in H^{2}(\Omega) \\
& \lesssim h_{k}^{2} \inf _{\substack{p_{1} \in H^{1}(\Omega) \\
p_{2} \in H^{2}(\Omega) \\
p_{1}+p_{2}=p}}\left\|p_{1}\right\|_{H^{1}(\Omega)}^{2}+\left\|p_{2}\right\|_{\beta^{-1} H^{2}(\Omega)}^{2}=h_{k}^{2}\|p\|_{H^{1}(\Omega)+\beta^{-1} H^{2}(\Omega)}^{2},
\end{aligned}
$$

for all $p \in H^{1}(\Omega) \cap L_{0}^{2}(\Omega)$, which finishes the proof. $\square$

Proof of Lemma 5.5. Let $\mathcal{F}(\tilde{u}, \tilde{p}):=(f, \tilde{u})_{L^{2}(\Omega)}+(g, \tilde{p})_{L^{2}(\Omega)}$ for $f \in\left[L^{2}(\Omega)\right]^{2}$ and $g \in H_{0}^{1}(\Omega) \cap L_{0}^{2}(\Omega)$. Choose $f_{2} \in\left[H_{0}^{1}(\Omega)\right]^{2}$ arbitrarily and define $f_{1}:=f-f_{2} \in$ $\left[L^{2}(\Omega)\right]^{2}$. Observe that

$$
\left(p_{\mathcal{F}}, \nabla \cdot \tilde{u}\right)_{L^{2}(\Omega)}=-\left(\nabla u_{\mathcal{F}}, \nabla \tilde{u}\right)_{L^{2}(\Omega)}-\beta\left(u_{\mathcal{F}}, \tilde{u}\right)_{L^{2}(\Omega)}+\left(f_{1}+f_{2}, \tilde{u}\right)_{L^{2}(\Omega)}
$$

is satisfied for all $\tilde{u} \in\left[H_{0}^{1}(\Omega)\right]^{2}$. This is equivalent to

$$
-\left(\nabla p_{\mathcal{F}}, \tilde{u}\right)_{L^{2}(\Omega)}=\left(\Delta u_{\mathcal{F}}, \tilde{u}\right)_{L^{2}(\Omega)}-\beta\left(u_{\mathcal{F}}, \tilde{u}\right)_{L^{2}(\Omega)}+\left(f_{1}+f_{2}, \tilde{u}\right)_{L^{2}(\Omega)}
$$

for all $\tilde{u} \in\left[H_{0}^{1}(\Omega)\right]^{2}$. Note that $\nabla p_{\mathcal{F}}, \Delta u_{\mathcal{F}}, u_{\mathcal{F}}, f_{1}$ and $f_{2}$ are in $\left[L^{2}(\Omega)\right]^{2}$. Therefore, the above statement holds for all $\tilde{u} \in\left[L^{2}(\Omega)\right]^{2}$ (because $\left[H_{0}^{1}(\Omega)\right]^{2}$ is dense in $\left[L^{2}(\Omega)\right]^{2}$ ), particularly for $\tilde{u}:=\nabla \tilde{p}$, where $\tilde{p} \in H^{1}(\Omega) \cap L_{0}^{2}(\Omega)$. So, we obtain

$$
-\left(\nabla p_{\mathcal{F}}, \nabla \tilde{p}\right)_{L^{2}(\Omega)}=\left(\Delta u_{\mathcal{F}}, \nabla \tilde{p}\right)_{L^{2}(\Omega)}-\beta\left(u_{\mathcal{F}}, \nabla \tilde{p}\right)_{L^{2}(\Omega)}+\left(f_{1}+f_{2}, \nabla \tilde{p}\right)_{L^{2}(\Omega)}
$$

for all $\tilde{p} \in H^{1}(\Omega) \cap L_{0}^{2}(\Omega)$.

Let $p_{1} \in H^{1}(\Omega) \cap L_{0}^{2}(\Omega)$ be such that

$$
-\left(\nabla p_{1}, \nabla \tilde{p}\right)_{L^{2}(\Omega)}=\left(\Delta u_{\mathcal{F}}, \nabla \tilde{p}\right)_{L^{2}(\Omega)}+\left(f_{1}, \nabla \tilde{p}\right)_{L^{2}(\Omega)}
$$

holds for all $\tilde{p} \in H^{1}(\Omega) \cap L_{0}^{2}(\Omega)$.

Note that $\Delta u_{\mathcal{F}}+f_{1} \in\left[L^{2}(\Omega)\right]^{2}$ and therefore the right-hand-side is a functional in $\left[H_{0}^{1}(\Omega)\right]^{*}$. So, existence and uniqueness of $p_{1} \in H^{1}(\Omega) \cap L_{0}^{2}(\Omega)$ is guaranteed. Using the choice $\tilde{p}:=p_{1}$, we obtain

$$
\left\|\nabla p_{1}\right\|_{L^{2}(\Omega)} \leq\left\|\Delta u_{\mathcal{F}}\right\|_{L^{2}(\Omega)}+\left\|f_{1}\right\|_{L^{2}(\Omega)} \leq\left\|u_{\mathcal{F}}\right\|_{H^{2}(\Omega)}+\left\|f_{1}\right\|_{L^{2}(\Omega)} .
$$

Using Poincaré's inequality, we obtain further

$$
\left\|p_{1}\right\|_{H^{1}(\Omega)} \lesssim\left\|u_{\mathcal{F}}\right\|_{H^{2}(\Omega)}+\left\|f_{1}\right\|_{L^{2}(\Omega)} .
$$

Let $p_{2} \in H^{1}(\Omega) \cap L_{0}^{2}(\Omega)$ be such that

$$
-\left(\nabla p_{2}, \nabla \tilde{p}\right)_{L^{2}(\Omega)}=-\beta\left(u_{\mathcal{F}}, \nabla \tilde{p}\right)_{L^{2}(\Omega)}+\left(f_{2}, \nabla \tilde{p}\right)_{L^{2}(\Omega)}
$$


for all $\tilde{p} \in H^{1}(\Omega) \cap L_{0}^{2}(\Omega)$. This implies, as $u_{\mathcal{F}} \in\left[H_{0}^{1}(\Omega)\right]^{2}$ and $f_{2} \in\left[H_{0}^{1}(\Omega)\right]^{2}$, that

$$
-\left(\nabla p_{2}, \nabla \tilde{p}\right)_{L^{2}(\Omega)}=\beta\left(\nabla \cdot u_{\mathcal{F}}, \tilde{p}\right)_{L^{2}(\Omega)}-\left(\nabla \cdot f_{2}, \tilde{p}\right)_{L^{2}(\Omega)}
$$

holds. As $\beta \nabla \cdot u_{\mathcal{F}}-\nabla \cdot f_{2} \in L^{2}(\Omega)$, existence and uniqueness of $p_{2}$ is guaranteed. Condition (R1) implies moreover $p_{2} \in H^{2}(\Omega)$ and

$$
\left\|p_{2}\right\|_{H^{2}(\Omega)}^{2} \lesssim \beta^{2}\left\|\nabla \cdot u_{\mathcal{F}}\right\|_{L^{2}(\Omega)}^{2}+\left\|\nabla \cdot f_{2}\right\|_{L^{2}(\Omega)}^{2} \lesssim \beta^{2}\left\|u_{\mathcal{F}}\right\|_{H^{1}(\Omega)}^{2}+\left\|f_{2}\right\|_{H^{1}(\Omega)}^{2} .
$$

Note that from 8.1), (8.2) and 8.4), we obtain

$$
\left(\nabla\left(p_{1}+p_{2}\right), \nabla \tilde{p}\right)_{L^{2}(\Omega)}=\left(\nabla p_{\mathcal{F}}, \nabla \tilde{p}\right)_{L^{2}(\Omega)}
$$

is satisfied for all $\tilde{p} \in H^{1}(\Omega) \cap L_{0}^{2}(\Omega)$, which implies (because $p_{\mathcal{F}} \in L_{0}^{2}(\Omega)$ and $p_{1}+p_{2} \in$ $\left.L_{0}^{2}(\Omega)\right)$ that $p_{\mathcal{F}}=p_{1}+p_{2}$ is satisfied. So, we have using (8.3) and (8.5)

$$
\begin{aligned}
\left\|p_{\mathcal{F}}\right\|_{P_{+, k}}^{2}=h_{k}^{2}\left\|p_{\mathcal{F}}\right\|_{H^{1}(\Omega)+\beta^{-1} H^{2}(\Omega)}^{2} & \quad \inf _{\substack{p=q_{1}+q_{2}, q_{1} \in H^{1}(\Omega) \cap L_{0}^{2}(\Omega)}} h_{k}^{2}\left\|q_{1}\right\|_{H^{1}(\Omega)}^{2}+h_{k}^{2}\left\|q_{2}\right\|_{\beta^{-1} H^{2}(\Omega)}^{2} \\
& q_{2} \in H^{2}(\Omega) \cap L_{0}^{2}(\Omega) \\
\leq & h_{k}^{2}\left\|p_{1}\right\|_{H^{1}(\Omega)}^{2}+h_{k}^{2}\left\|p_{2}\right\|_{\beta^{-1} H^{2}(\Omega)}^{2} \\
& \lesssim h_{k}^{2}\left\|u_{\mathcal{F}}\right\|_{H^{2}(\Omega)}^{2}+h_{k}^{2}\left\|u_{\mathcal{F}}\right\|_{\beta H^{1}(\Omega)}^{2}+h_{k}^{2}\left\|f_{1}\right\|_{L^{2}(\Omega)}^{2}+h_{k}^{2}\left\|f_{2}\right\|_{\beta^{-1} H^{1}(\Omega)}^{2} \\
& =h_{k}^{2}\left\|u_{\mathcal{F}}\right\|_{H^{2}(\Omega) \cap \beta H^{1}(\Omega)}^{2}+h_{k}^{2}\left\|f_{1}\right\|_{L^{2}(\Omega)}^{2}+h_{k}^{2}\left\|f_{2}\right\|_{\beta^{-1} H^{1}(\Omega)}^{2} .
\end{aligned}
$$

As $f_{2} \in\left[H_{0}^{1}(\Omega)\right]^{2}$ was chosen arbitrarily, we can take the infimum over all $f_{2}$, which finishes the proof.

Proof of Lemma 5.6. As $H_{0}^{1}(\Omega)$ is dense in $L^{2}(\Omega)$, for $u \in\left[H^{2}(\Omega)\right]^{2}$ the function $-\Delta u \in\left[L^{2}(\Omega)\right]^{2}$ can be approximated by some function $w^{\epsilon} \in\left[H_{0}^{1}(\Omega)\right]^{2}$ such that $\left\|(-\Delta) u-w^{\epsilon}\right\|_{L^{2}(\Omega)}^{2} \leq \epsilon$. So, we can introduce an operator $-\Delta^{\epsilon}:\left[H^{2}(\Omega)\right]^{2} \rightarrow$ $\left[H_{0}^{1}(\Omega)\right]^{2}$, which approximates $-\Delta u$ in $\left[H_{0}^{1}(\Omega)\right]^{2}$, satisfying the error estimate

$$
\left\|(-\Delta) u-\left(-\Delta^{\epsilon}\right) u\right\|_{L^{2}(\Omega)}^{2} \leq \epsilon
$$

for all $u \in H^{2}(\Omega)$. So, $-\Delta^{\epsilon} u$ approaches $-\Delta u$ in the $L^{2}$-sense for $\epsilon \rightarrow 0$. (As $-\Delta u \notin\left[H_{0}^{1}(\Omega)\right]^{2}$ in general, $\left\|-\Delta^{\epsilon} u\right\|_{H^{1}(\Omega)}$ is not convergent for $\epsilon \rightarrow 0$.)

Analogously, we introduce the operator $\nabla^{\epsilon}: H^{1}(\Omega) \rightarrow\left[H_{0}^{1}(\Omega)\right]^{2}$, which approximates $\nabla p$ in $\left[H_{0}^{1}(\Omega)\right]^{2}$, satisfying the error estimate

$$
\left\|\nabla p-\nabla^{\epsilon} p\right\|_{L^{2}(\Omega)}^{2} \leq \epsilon
$$

for all $p \in H^{1}(\Omega)$. So, $\nabla^{\epsilon} p$ approaches $\nabla p$ in the $L^{2}$-sense for $\epsilon \rightarrow 0$. (As $\nabla p \notin$ $\left[H_{0}^{1}(\Omega)\right]^{2}$ in general, $\left\|\nabla^{\epsilon} p\right\|_{H^{1}(\Omega)}$ is not convergent for $\epsilon \rightarrow 0$.)

The idea of this proof is to show that for all $\epsilon>0$ there is some $\tilde{x}^{\epsilon} \in X$ such that

$$
\begin{aligned}
\mathcal{B}\left(x_{\mathcal{F}}, \tilde{x}^{\epsilon}\right) & \gtrsim h_{k}^{-2}\left\|u_{\mathcal{F}}\right\|_{U_{+, k}}^{2}-\epsilon\left(1+\beta^{1 / 2}+\beta\right) h_{k}^{-1}\left\|x_{\mathcal{F}}\right\|_{X_{+, k}}-\epsilon^{2} \text { and } \\
\mathcal{F}\left(\tilde{x}^{\epsilon}\right) & \lesssim h_{k}^{-2}\|\mathcal{F}\|_{\left[X_{-, k}\right]^{*}}\left\|x_{\mathcal{F}}\right\|_{X_{+, k}}+\epsilon\left(1+\beta^{1 / 2}\right) h_{k}^{-1}\|\mathcal{F}\|_{\left(X_{-, k}\right)^{*}}+\epsilon^{2}
\end{aligned}
$$

holds. As $\mathcal{B}\left(x_{\mathcal{F}}, \tilde{x}^{\epsilon}\right)=\mathcal{F}\left(\tilde{x}^{\epsilon}\right)$, the statement of the Lemma follows for $\epsilon \rightarrow 0$. 
In the following, we show (8.6) and 8.7) for $\tilde{x}^{\epsilon}:=\left(-\Delta^{\epsilon} u_{\mathcal{F}}, \nabla \cdot \nabla^{\epsilon} p_{\mathcal{F}}\right)$. First, we show (8.6). Here, we estimate the summands of $\mathcal{B}\left(x_{\mathcal{F}}, \tilde{x}^{\epsilon}\right)$ separately. We have

$$
\begin{aligned}
& \left(\nabla u_{\mathcal{F}}, \nabla\left(-\Delta^{\epsilon}\right) u_{\mathcal{F}}\right)_{L^{2}(\Omega)}=\left(\Delta u_{\mathcal{F}}, \Delta^{\epsilon} u_{\mathcal{F}}\right)_{L^{2}(\Omega)} \\
& \quad \geq\left(\Delta u_{\mathcal{F}}, \Delta u_{\mathcal{F}}\right)_{L^{2}(\Omega)}-\epsilon\left\|\Delta u_{\mathcal{F}}\right\|_{L^{2}(\Omega)} \geq\left\|u_{\mathcal{F}}\right\|_{H^{2}(\Omega)}^{2}-\epsilon \beta^{1 / 2} h_{k}^{-1}\left\|x_{\mathcal{F}}\right\|_{X_{+, k}},
\end{aligned}
$$

where we use for the first step that $\Delta^{\epsilon}$ maps into $H_{0}^{1}(\Omega)$. For the second step, we use the Cauchy-Schwarz inequality and the upper bound for $\left\|-\Delta u_{\mathcal{F}}-\left(-\Delta^{\epsilon}\right) u_{\mathcal{F}}\right\|_{L^{2}(\Omega)}$. Friedrichs' inequality is used for the third step. Using similar arguments, we obtain for the next summand

$$
\begin{aligned}
& \beta\left(u_{\mathcal{F}},-\Delta^{\epsilon} u_{\mathcal{F}}\right)_{L^{2}(\Omega)} \geq \beta\left(u_{\mathcal{F}},-\Delta u_{\mathcal{F}}\right)_{L^{2}(\Omega)}-\epsilon \beta\left\|u_{\mathcal{F}}\right\|_{L^{2}(\Omega)} \\
& \quad=\beta\left(\nabla u_{\mathcal{F}}, \nabla u_{\mathcal{F}}\right)_{L^{2}(\Omega)}-\epsilon \beta\left\|u_{\mathcal{F}}\right\|_{L^{2}(\Omega)} \gtrsim \beta\left\|u_{\mathcal{F}}\right\|_{H^{1}(\Omega)}^{2}-\epsilon \beta h_{k}^{-1}\left\|x_{\mathcal{F}}\right\|_{X_{+, k}} .
\end{aligned}
$$

We obtain for the last two summands, corresponding to the (2,1)- and the (1,2)-block, again using similar arguments, including the bound for $\left\|\nabla p_{\mathcal{F}}-\nabla^{\epsilon} p_{\mathcal{F}}\right\|_{L^{2}(\Omega)}$, that

$$
\begin{aligned}
& \left(\nabla \cdot u_{\mathcal{F}}, \nabla \cdot \nabla^{\epsilon} p_{\mathcal{F}}\right)_{L^{2}(\Omega)}+\left(\nabla \cdot\left(-\Delta^{\epsilon}\right) u_{\mathcal{F}}, p_{\mathcal{F}}\right)_{L^{2}(\Omega)} \\
& \quad=-\left(\nabla \nabla \cdot u_{\mathcal{F}}, \nabla^{\epsilon} p_{\mathcal{F}}\right)_{L^{2}(\Omega)}+\left(\Delta^{\epsilon} u_{\mathcal{F}}, \nabla p_{\mathcal{F}}\right)_{L^{2}(\Omega)} \\
& \quad \geq-\left(\nabla \nabla \cdot u_{\mathcal{F}}, \nabla^{\epsilon} p_{\mathcal{F}}\right)_{L^{2}(\Omega)}+\left(\Delta^{\epsilon} u_{\mathcal{F}}, \nabla^{\epsilon} p_{\mathcal{F}}\right)_{L^{2}(\Omega)}-\epsilon\left\|\Delta^{\epsilon} u_{\mathcal{F}}\right\|_{L^{2}(\Omega)}
\end{aligned}
$$

holds. Using integration by parts, the Cauchy-Schwarz inequality and the upper bound for $\left\|-\Delta u_{\mathcal{F}}-\left(-\Delta^{\epsilon}\right) u_{\mathcal{F}}\right\|_{L^{2}(\Omega)}$, we obtain further

$$
\begin{aligned}
& \left(\nabla \cdot u_{\mathcal{F}}, \nabla \cdot \nabla^{\epsilon} p_{\mathcal{F}}\right)_{L^{2}(\Omega)}+\left(\nabla \cdot\left(-\Delta^{\epsilon}\right) u_{\mathcal{F}}, p_{\mathcal{F}}\right)_{L^{2}(\Omega)} \\
& \quad \geq\left(\nabla u_{\mathcal{F}}, \nabla \nabla^{\epsilon} p_{\mathcal{F}}\right)_{L^{2}}+\left(\Delta u_{\mathcal{F}}, \nabla^{\epsilon} p_{\mathcal{F}}\right)_{L^{2}}-\epsilon\left(\left\|\Delta u_{\mathcal{F}}\right\|_{L^{2}}+\left\|\nabla^{\epsilon} p_{\mathcal{F}}\right\|_{L^{2}}+\epsilon\right) \\
& \quad=\underbrace{\left(\nabla u_{\mathcal{F}}, \nabla \nabla^{\epsilon} p_{\mathcal{F}}\right)_{L^{2}}-\left(\nabla u_{\mathcal{F}}, \nabla \nabla^{\epsilon} p_{\mathcal{F}}\right)_{L^{2}}}_{=0}-\epsilon\left(\left\|\Delta u_{\mathcal{F}}\right\|_{L^{2}}+\left\|\nabla^{\epsilon} p_{\mathcal{F}}\right\|_{L^{2}}+\epsilon\right) .
\end{aligned}
$$

Finally, we obtain using the upper bound for $\left\|\nabla p_{\mathcal{F}}-\nabla^{\epsilon} p_{\mathcal{F}}\right\|_{L^{2}(\Omega)}$ and the definition of $\|\cdot\|_{X_{+, k}}$ that

$$
\begin{aligned}
& \left(\nabla \cdot u_{\mathcal{F}}, \nabla \cdot \nabla^{\epsilon} p_{\mathcal{F}}\right)_{L^{2}(\Omega)}+\left(\nabla \cdot\left(-\Delta^{\epsilon}\right) u_{\mathcal{F}}, p_{\mathcal{F}}\right)_{L^{2}(\Omega)} \\
& \quad \geq-\epsilon\left(\left\|\Delta u_{\mathcal{F}}\right\|_{L^{2}(\Omega)}+\left\|\nabla p_{\mathcal{F}}\right\|_{L^{2}(\Omega)}+\epsilon\right) \geq-\epsilon\left(\left\|u_{\mathcal{F}}\right\|_{H^{2}(\Omega)}+\left\|p_{\mathcal{F}}\right\|_{H^{1}(\Omega)}+\epsilon\right) \\
& \quad \geq-\epsilon\left(1+\beta^{1 / 2}\right) h_{k}^{-1}\left\|x_{\mathcal{F}}\right\|_{X_{+, k}}-\epsilon^{2}
\end{aligned}
$$

holds. So, we have shown 8.6). The next step is to show 8.7). Let $\mathcal{F}(\tilde{u}, \tilde{p}):=$ $(f, \tilde{u})_{L^{2}(\Omega)}+(g, \tilde{p})_{L^{2}(\Omega)}$ for $f \in\left[L^{2}(\Omega)\right]^{2}$ and $g \in H_{0}^{1}(\Omega) \cap L_{0}^{2}(\Omega)$. Let $f_{2} \in\left[H_{0}^{1}(\Omega)\right]^{2}$ and $f_{1}:=f-f_{2}$. Using the same arguments as above,

$$
\left(f_{1},-\Delta^{\epsilon} u_{\mathcal{F}}\right)_{L^{2}(\Omega)} \lesssim\left\|f_{1}\right\|_{L^{2}(\Omega)}\left\|u_{\mathcal{F}}\right\|_{H^{2}(\Omega)}+\epsilon\left\|f_{1}\right\|_{L^{2}(\Omega)}
$$

holds as well as

$$
\begin{aligned}
& \left(f_{2},-\Delta^{\epsilon} u_{\mathcal{F}}\right)_{L^{2}(\Omega)} \lesssim\left(\nabla f_{2}, \nabla u_{\mathcal{F}}\right)_{L^{2}(\Omega)}+\epsilon\left\|f_{2}\right\|_{L^{2}(\Omega)} \\
& \quad \lesssim\left\|f_{2}\right\|_{\beta^{-1} H^{1}(\Omega)}\left\|u_{\mathcal{F}}\right\|_{\beta H^{1}(\Omega)}+\epsilon \beta^{1 / 2}\left\|f_{2}\right\|_{\beta^{-1} H^{1}(\Omega)} .
\end{aligned}
$$

This implies

$$
\begin{aligned}
(f,- & \left.\Delta^{\epsilon} u_{\mathcal{F}}\right)_{L^{2}(\Omega)} \\
& \lesssim\|f\|_{L^{2}(\Omega)+\beta^{-1} H_{0}^{1}(\Omega)}\left\|u_{\mathcal{F}}\right\|_{H^{2}(\Omega) \cap \beta H^{1}(\Omega)}+\epsilon\left(1+\beta^{1 / 2}\right)\|f\|_{L^{2}(\Omega)+\beta^{-1} H_{0}^{1}(\Omega)} \\
& \lesssim\|f\|_{L^{2}(\Omega)+\beta^{-1} H_{0}^{1}(\Omega)}\left\|u_{\mathcal{F}}\right\|_{H^{2}(\Omega) \cap \beta H^{1}(\Omega)}+\epsilon h_{k}^{-1}\left(1+\beta^{1 / 2}\right)\|\mathcal{F}\|_{\left[X_{-, k}\right]^{*}}
\end{aligned}
$$


Let $p_{2} \in H^{2}(\Omega)$ and $p_{1}:=p-p_{2}$. We have

$$
\left(g, \nabla \cdot \nabla^{\epsilon} p_{1}\right)_{L^{2}(\Omega)}=-\left(\nabla g, \nabla^{\epsilon} p_{1}\right)_{L^{2}(\Omega)} \lesssim\|g\|_{H^{1}(\Omega)}\left\|p_{1}\right\|_{H^{1}(\Omega)}+\epsilon\|g\|_{H^{1}(\Omega)} .
$$

Using $g \in H_{0}^{1}(\Omega)$, we have also

$$
\begin{aligned}
& \left(g, \nabla \cdot \nabla^{\epsilon} p_{2}\right)_{L^{2}(\Omega)}=-\left(\nabla g, \nabla^{\epsilon} p_{2}\right)_{L^{2}(\Omega)} \lesssim-\left(\nabla g, \nabla p_{2}\right)_{L^{2}(\Omega)}+\epsilon\|g\|_{H^{1}(\Omega)} \\
& \quad=\left(g, \nabla \cdot \nabla p_{2}\right)_{L^{2}(\Omega)}+\epsilon\|g\|_{H^{1}(\Omega)} \leq\|g\|_{\beta L^{2}(\Omega)}\left\|p_{1}\right\|_{\beta^{-1} H^{2}(\Omega)}+\epsilon\|g\|_{H^{1}(\Omega)}
\end{aligned}
$$

and, by combining these estimates,

$$
\begin{aligned}
\left(g, \nabla \cdot \nabla^{\epsilon} p_{\mathcal{F}}\right)_{L^{2}(\Omega)} & \lesssim\|g\|_{H_{0}^{1}(\Omega)+\beta L_{0}^{2}(\Omega)}\left\|p_{\mathcal{F}}\right\|_{H^{1}(\Omega)+\beta^{-1} H^{2}(\Omega)}+\epsilon\|g\|_{H^{1}(\Omega)} \\
& \lesssim\|g\|_{H_{0}^{1}(\Omega)+\beta L_{0}^{2}(\Omega)}\left\|p_{\mathcal{F}}\right\|_{H^{1}(\Omega)+\beta^{-1} H^{2}(\Omega)}+\epsilon h_{k}^{-1}\|\mathcal{F}\|_{\left(X_{-, k}\right)^{*}}
\end{aligned}
$$

By combining these results, we immediately obtain 8.7 . This finishes the proof. $\square$

Proof of inequality 5.4. First note that

$$
\begin{aligned}
& \left\|\psi_{k} p_{k}\right\|_{H^{1}(\Omega)} \approx\left\|\nabla\left(\psi_{k} p_{k}\right)\right\|_{L^{2}(\Omega)}+\left\|\psi_{k} p_{k}\right\|_{L^{2}(\Omega)} \\
& \quad \lesssim\left\|\left(\nabla \psi_{k}\right) p_{k}\right\|_{L^{2}(\Omega)}+\left\|\psi_{k} \nabla p_{k}\right\|_{L^{2}(\Omega)}+\left\|\psi_{k} p_{k}\right\|_{L^{2}(\Omega)} \\
& \quad \leq\left\|\nabla \psi_{k}\right\|_{L^{\infty}(\Omega)}\left\|p_{k}\right\|_{L^{2}(\Omega)}+\left\|\psi_{k}\right\|_{L^{\infty}(\Omega)}\left\|\nabla p_{k}\right\|_{L^{2}(\Omega)}+\left\|\psi_{k}\right\|_{L^{\infty}(\Omega)}\left\|p_{k}\right\|_{L^{2}(\Omega)}
\end{aligned}
$$

holds due to the product rule and Hölder's inequality. Here, $\|\cdot\|_{L^{\infty}(\Omega)}$ is the standard $L^{\infty}$-norm (essential supremum). As $\psi_{k}(\xi)$ is piecewise linear and $\psi_{k}(\xi) \in[0,1]$, $\left|\nabla \psi_{k}(\xi)\right| \leq h_{k}^{-1}$ holds for almost all $\xi \in \Omega$. Using this, $\left|\psi_{k}(\xi)\right| \leq 1$ and a standard inverse inequality for estimating $\left\|\nabla p_{k}\right\|_{L^{2}(\Omega)}$ from above, one obtains $\left\|\psi_{k} p_{k}\right\|_{H^{1}(\Omega)} \lesssim$ $\left(1+h_{k}^{-1}\right)\left\|p_{k}\right\|_{L^{2}(\Omega)}$ and, assuming $h_{k} \lesssim 1$, the desired result.

Proof of inequality (5.5). Assume that the mesh consists of the elements $\mathcal{T}_{i}$ for $i=1, \ldots, M_{k}$. Note that it suffices to show 5.5 for each element $\mathcal{T}_{i}$ or that

$$
\left(p_{k}, p_{k}\right)_{L^{2}\left(\mathcal{T}_{i}\right)} \leq C\left(p_{k}, \psi_{k} p_{k}\right)_{L^{2}\left(\mathcal{T}_{i}\right)}
$$

for all $p_{k} \in \hat{P}_{k}$. Note that $\psi_{k}=1$ on all interior elements (no vertex is located on $\partial \Omega$ ), so $(8.8)$ holds with $C=1$ in this case. So, it remains to show the inequality for the elements elements where one (case 1) or two vertices (case 2) are located on $\partial \Omega$. (Note that by assumption each element has at least one vertex in the interior of $\Omega$ ).

Note that, both $p_{k}$ and $\psi_{k}$, are linear functions. The integrals in 8.8 can be computed using the reference element $\Delta:=\left\{\left(\xi_{1}, \xi_{2}\right) \in(0,1)^{2}: \xi_{1}+\xi_{2}<1\right\}$. Using the linear transformation $\Phi_{i}: \Delta \rightarrow \mathcal{T}_{i}$, the substitution rule states that 8.8 is equivalent to

$$
\left|\operatorname{det} \nabla \Phi_{i}\right|\left(\hat{p}_{k}, \hat{p}_{k}\right)_{L^{2}\left(\mathcal{T}_{i}\right)} \leq C\left|\operatorname{det} \nabla \Phi_{i}\right|\left(\hat{p}_{k}, \hat{\psi}_{k} \hat{p}_{k}\right)_{L^{2}\left(\mathcal{T}_{i}\right)},
$$

where $\hat{p}(\xi)=u\left(\Phi_{i}^{-1}(\xi)\right)$ and $\hat{\psi}(\xi)=\psi\left(\Phi_{i}^{-1}(\xi)\right)$. Here, $\left|\operatorname{det} \nabla \Phi_{i}\right|$ is the absolute value of the Jacobi determinant of the transformation. As this is a positive constant, it can be canceled out.

For the case 1 , the function $\hat{\psi}_{k}$ takes the value 1 on two vertices and the value 0 on one vertex. We assume without loss of generality, that it takes the value 0 on $(0,1)$. So, we obtain $\hat{\psi}_{k}(\xi)=1-\xi_{2}$. Using the ansatz $\hat{p}_{k}\left(\xi_{1}, \xi_{2}\right):=a_{0}+a_{1} \xi_{1}+a_{2} \xi_{2}$, we can compute both integrals in 8.9 and obtain that 8.9 holds for $C=\frac{1}{3}(6+\sqrt{6})$.

For the case 2 , the function $\hat{\psi}_{k}$ takes the value 0 on two vertices and the value 1 on one vertex. We assume without loss of generality that it takes the value 1 on the vertex $(0,1)$. So, we obtain $\hat{\psi}_{k}(\xi)=\xi_{2}$. Here we obtain that 8.9 holds for $C=(4+\sqrt{6})$. This finishes the proof. 


\section{REFERENCES}

[1] R. Adams and J. Fournier, Sobolev Spaces, 2nd ed, Academic Press (2008).

[2] M. Bercovier and O. Pironneau, Error estimates for finite element method solution of the Stokes problem in primitive variables, Numerische Mathematik 33, p. $211-224$ (1979).

[3] J. Bergh and J. Löfström, Interpolation Spaces, an Introduction, Springer, Berlin (1976).

[4] D. Braess and R. Sarazin, An efficient smoother for the Stokes problem, Applied Numerical Mathematics 23, p. 3 - 19 (1997).

[5] J.H Bramble and J.E. Pasciak, Iterative techniques for time dependent stokes problems, Comp. Math. Appl. 33, p. 13 - 30 (1997).

[6] S. Brenner, A nonconforming multigrid method for the stationary stokes problem, Mathematics of Computation 55, p. 411 - 473 (1990).

[7] S.C. Brenner, Multigrid methods for parameter dependent problems, RAIRO, Modélisation Math. Anal. Numér 30, p. 265 - 297 (1996).

[8] M. Dauge, Elliptic boundary value problems on corner domains. Smoothness and asymptotics of solutions, Lecture Notes in Mathematics, Springer, Berlin (1988).

[9] W. Hackbusch, Multi-Grid Methods and Applications, Springer, Berlin (1985).

[10] R.B. Kellogg and J.E Osborn, A regularity result for the Stokes problem in a convex polygon, Journal of Functional Analysis 21, p. 397 - 431 (1976).

[11] G. M. Kobelkov and M. A. Olshanskii, Effective preconditioning of uzawa type schemes for generalized stokes problem, Numerische Mathematik 86, p. $443-470$ (2000).

[12] M. Larin and A. Reusken, A comparative study of efficient iterative solvers for generalized stokes problem, Numer. Linear Algebra Appl. 15, p. 13 - 34 (2008).

[13] K. Mardal and R. Winther, Uniform preconditioners for the time dependent stokes problem, Numerische Mathematik 98, p. 305 - 327 (2004).

[14] _ Uniform preconditioners for the time dependent stokes problem (erratum), Numerische Mathematik 103, p. $171-172$ (2006).

[15] M. Olshanskii, Multigrid Analysis for the Time Dependent Stokes Problem, Mathematics of Computation 81, p. $57-79$ (2012).

[16] M. Olshanskii, J. Peters, and A. Reusken, Uniform preconditioners for a parameter dependent saddle point problem with application to generalized stokes interface equations, Numerische Mathematik 105, p. 159 - 191 (2006).

[17] Verführth R., A multilevel algorithm for mixed problems, SIAM J. on Numerical Analysis 21, p. $264-271$ (1984).

[18] J. Schöberl and W. Zulehner, On Schwarz-type Smoothers for Saddle Point Problems, Numerische Mathematik 95, p. 3777 - 399 (2002).

[19] S. Takacs, A robust all-at-once multigrid method for the Stokes control problem, Numerische Mathematik 130, p. $517-540$ (2015).

[20] S. Takacs and W. Zulehner, Convergence analysis of all-at-once multigrid methods for elliptic control problems under partial elliptic regularity, SIAM J. on Numerical Analysis 51 (2013) p. $1853-1874$.

[21] S. P. Vanka, Block-implicit multigrid solution of Navier-Stokes equations in primitive variables, Mathematica of Computation 65, p. 138 - 158 (1986).

[22] R. Verfürth, Error estimates for a mixed finite element approximation of the Stokes equations, RAIRO 18, p. 175 - 182 (1984).

[23] P. Wesseling and C. W. Oosterlee, Geometric multigrid with applications to computational fluid dynamics, J. Comput. Appl. Math. 128, p. 311 - 334 (2001).

[24] W. Zulehner, A class of smoothers for saddle point problems, Computing. 65, p. $227-246$ (2000).

[25] _ Non-standard Norms and Robust Estimates for Saddle Point Problems, SIAM J. on Matrix Anal. \& Appl 32, p. 536 - 560 (2011).

First Published in SINUM in 53 (6), p. $634-2654,2015$, published by the Society of Industrial and Applied Mathematics (SIAM) http://epubs.siam.org/doi/10.1137/140969658

Copyright by SIAM. Unauthorized reproduction of this article is prohibited. 